\title{
On the study of a singular point of Briot-Bouquet type of a system of ordinary nonlinear differential equations
}

By

Masahiro IWANO*

\section{Introduction.}

In this note we consider a system of ordinary nonlinear differential equations which, in vectorial form, can be written

$$
x y^{\prime}=f(x, y) \quad\left(\prime^{\prime}=\frac{d}{d x}\right) .
$$

Here:

i) $x$ is a complex variable.

ii) $y$ is a column vector of n components.

iii) $f(x, y)$ is an $n$-dimensional column vector function of $x$ and $y$, holomorphic and bounded in a domain$$
|x|<a, \quad\|y\|<b
$$$$
\left(\|y\|=\max _{j=1}^{n}\left|y_{j}\right|\right)
$$

and vanishing at $(0,0), y_{j}$ being the $j^{\text {th }}$ component of $y$.

The singular points $x=0$ of the equation (A) are usually said to be of Briot-Bouquet type. These singular points have been studied by diverse authors since $\mathrm{C}$. Briot and J. Bouquet. The present author is specially interested in the case when the eigenvalues of the matrix $F \stackrel{\prime}{=} f_{y}(0,0)$ are all zero.

$\mathbf{1}^{\circ}$. In 1937, M. Hukuhara [1] studied first the case for $n=1$. In the case when $n \geqq 2$, some difficulty comes up in the step of a formal

Received June 29, 1966.

Communicated by M. Hukuhara.

* Sponsored by the Mathematics Research Center, United States Army, University of Wisconsin, under Contract No.: DA-11-022-ORD-2059. 
transformation. After twelve years, M. Hukuhara [2] discussed about the construction of formal solutions of the equation (A) for the case when the vector $f(x, y)$ has two components of the form

$$
f(x, y)=y^{\mathscr{M}} F(x, y) y, \quad\left(y^{\mathscr{M}}=y_{1}^{m_{1}} y_{2}^{m_{2}}\right),
$$

where $F(x, y)$ is a 2 -by-2 diagonal matrix and the matrix $F(0,0)$ is nonsingular, $\mathscr{M}$ is a 2 -dimensional row vector with components $m_{1}$ and $m_{2}$ nonnegative integers not simultaneously zero.

Recently, the present author has studied an equation similar to Hukunara's i.e. the equation (A) for the case when the vector $f(x, y)$ has the following form, instead of (0.1),

$$
f(x, y)=y \mathscr{M H}_{F}(y) y+x g(x, y),
$$

where $F(y)$ is a 2-by-2 diagonal matrix and $g(x, y)$ is a 2-dimensional column vector.

He introduced the following assumption:

Let $\alpha$ be the 2-vector formed by the diagonal components of the matrix $F(0)$. Then, boin components of the 2-vector $\frac{1}{(\mathscr{M} \cdot \alpha)}$ a have positive real parts, where the symbol $(\cdot)$ denotes the inner product.

Under these assumptions, M. Iwano [2] obtained formal solutions of diverse types and investigated the analytical meaning of each of these formal solutions.

$2^{\circ}$. The results obtained in M. Iwano [2] can be extended to the case for $n>2$ if we introduce the following assumptions similar to those in the case of $n=2$ :

iv) The n-vector function $f(x, y)$ admits the uniformly convergent expansion

$$
f(x, y)=y \mathscr{M} \mathbb{1}_{n}\left(f^{(0)}(y)\right) y+\sum_{k=1}^{\infty} f^{(k)}(y) x^{k}
$$

$$
y^{\mathscr{M}}=y_{1}^{m_{1}} y_{2}^{m_{2}} \ldots y_{n}^{m_{n}},
$$

for $(x, y)$ in the domain (A.1), where $\mathbf{1}_{n}(y)$ is an $n$-by-n diagonal matrix such that the n-vector formed by its diagonal components 
coinciac's with ihe n-recior $y$, the n-vectors $f^{(k)}(y)(k \geqq 0)$ are functions holomo phic and bounded in $y$ for

$$
\|y\|<b
$$

$\mathcal{M}$ is an n-dimensional row vector whose components are nonnegative integers $m_{j}(j=1,2, \cdots, n)$ not simultaneously zero.

v) The n-vector $\frac{1}{(\mathcal{M} \cdot \alpha)} \alpha$, where

$$
\alpha=f^{(0)}(0)
$$

has $n$ components slich that their real parts are all positive.

Under the assumptions i), ii) $\sim \mathrm{v}$ ), the author proved the following theorem in the paper (M. Iwano [3]) titled "Convergent solution of ordinary nonlinear differential equations":

Theorern A. Suppose that all the assumptions i), ii) $\sim$ ) are sacisfied. Then, the equation (A) admits a solution $y=S(x, U)$ rith the properties that:

(a) The n-vector $S(x, u)$ is a function of $x$ and $u$ (the n-rector), holomorphic and bounded in a domain

$$
|x|<a^{\prime \prime}, \quad\|u\|<b^{\prime \prime}, \quad u \mathscr{M} \in \widetilde{\mathfrak{D}}\left(c^{\prime \prime}\right),
$$

where

(A. 7)

$$
\widetilde{\mathfrak{D}}\left(c^{\prime \prime}\right)=\left\{w: \Theta_{1}<\arg w<\Theta_{2}, 0<|w|<c^{\prime \prime}\right\}
$$

with

$$
\text { (A. 8) } \Theta_{1}=-\pi-\min _{j=1}^{n} \arg \alpha_{j}+4 \delta_{0}, \quad \Theta_{2}=\pi-\max _{j=1}^{n} \arg \alpha_{j}-4 \delta_{0} .
$$

$\delta_{0}$ being a sufficiently small positive constant. Moreover, $S(x, u)$ admits the uniformly convergent expansion

$$
S(x, u)=u+\mathbf{1}_{n}(u) P^{(0)}(u)+\sum_{k=1}^{\infty} P^{(k)}(u) x^{k}
$$

for the domain (A.6).

(b) The coefficient $P^{(0)}(u)$ is an n-dimensional column vector function holomorphic and bounded in $u$ for

$$
\|u\|<b^{\prime \prime}
$$


and developable there in a uniformly convergent power series of $u$ :
$(\mathrm{A} .10)^{0}$
$P^{(0)}(u)=\sum_{|\mathcal{K}| \geqq 1} P^{(0)} u^{\mathcal{K}}$,
$\left(|\mathcal{K}|=\sum_{j=1}^{n} k_{j}\right)$.

If $k \geqq 1$, the coefficients $P^{(k)}(u)$ are $n$-dimensional column vector functions holomorphic and bounded in $u$ for

$$
\|u\|<b^{\prime \prime}, \quad u \mathscr{M} \in \mathfrak{D}\left(c^{\prime \prime}\right) .
$$

and admitting the asymptotic expansions

$$
P^{(k)}(u) \simeq \sum_{\mathcal{K}} P_{\mathcal{K}}^{(k)} u^{\varkappa}
$$

as $u$ tends to 0 in the domain (A.11).

(c) $U \equiv U\left(x, x_{0}, u^{0}\right)$ is the holomorphic solution of a simplified equation satisfying the initial condition $U=u^{0}$ at $x=x_{0}$, where $\left(x_{0}, u^{0}\right)$ is an arbitrary point in the domain (A.6). The simplified equation has the form

$$
x u^{\prime}=u \mathscr{M} \mathbf{1}_{n}\left(\alpha+\sum_{\mathscr{H} \in \Phi^{\prime}} \beta_{\mathscr{H}} u \mathscr{H}\right) u .
$$

Here, $\mathfrak{S}$ is the set of vectors $\mathcal{K}$ for which we have

$$
((\mathcal{K}-\mathscr{M}) \cdot \alpha)=0 \text {, }
$$

and if the n-vector constant $\beta_{\mathscr{H}}$ is non-zero, we have

$$
\text { (A.13) } \quad\left((\mathcal{K}-\mathscr{M}) \cdot \beta_{\mathscr{H}}\right)=0 \quad \text { for any } \mathcal{K} \in \mathbb{S} \text {. }
$$

$3^{\circ}$. However, in the proof of the Theorem A, he assumed that the following two theorems have been established:

Theorem A.1. Under the same assumptions as in Theorem A, there exists a formal transformation of the form

$$
y \sim u+\mathbf{1}_{n}(u) P^{(0)}(u)+\sum_{k=1}^{\infty} P^{(k)}(u) x^{k},
$$

where the n-vectors $P^{(k)}(u)$ are formal power series of $u$ given by (A.10) ${ }^{k}$, such that the equation (A) is formally transformed into the equation ( $\mathrm{R})$.

Theorem A. 2. The n-vector function $P^{(0)}(U)$ can be uniquely 
determined as a solution of the non-linear differential equation

$$
\begin{gathered}
x P^{\prime}=U \mathscr{M}\left((\mathbf{1}+P) \mathscr{M} \mathbf{1}_{n}(\mathbf{1}+P) f^{(0)}\left(U+\mathbf{1}_{n}(U) P\right)\right. \\
\left.-\left(\alpha+\alpha^{*} U \mathscr{M}\right)-\mathbf{1}_{n}\left(\alpha+\alpha^{*} U \mathscr{M}\right) P\right), \\
\alpha^{*}=\sum_{\mathscr{H} \in \mathfrak{S}} \beta \mathscr{H}_{\mathcal{C}} U \mathscr{H}-\mathscr{M}
\end{gathered}
$$

in such a way that the n-vector $P^{(0)}(u)$ is a function holomorphic and bounded in $u$ for
(A. 16)
$\|u\|<b_{0}^{\prime \prime}$
$\left(0<b^{\prime \prime}<b_{0}^{\prime \prime}<b\right)$

and developable there in the uniformly convergent power series (A.10) ${ }^{0}$. Here, $\mathbf{1}$ is an n-dimensional column vector whose components are all equal to 1.

If $k \geqq 1$, the n-vector functions $P^{(k)}(U)$ can be uniquely determined as solutions of the linear differential equations

$(\mathrm{A} .17)^{k} \quad x P^{\prime}=-k P+F\left(U+\mathbb{1}_{n}(U) P^{(0)}(U)\right) P+R^{(k)}(U)$,

$$
F(y)=\frac{\partial}{\partial y}\left(y \mathbb{1}_{n}\left(f^{(0)}(y)\right) y\right), \quad F(0)=0
$$

in such a way that the n-vectors $P^{(k)}(u)$ are functions holomorphic, bounded and admitting the asymptotic expansions (A. 10) ${ }^{k}$ as $u$ tends to 0 in the domain

$$
\|u\|<b_{0}^{\prime \prime}, \quad u^{\mathscr{M}} \in \mathfrak{S}\left(c_{0}^{\prime \prime}\right), \quad\left(0<c^{\prime \prime} \leqq c_{0}^{\prime \prime}\right) .
$$

Here, the n-vector $R^{(k)}(u)$ is a known function admitting an asymptotic expansion in powers of $u$ as $u$ tends to 0 in the domain (A.19) if re'e assume that the functions $P^{(h)}(u)$ have been already determined for $h<k$.

Therefore, for the complete proof of the Theorem A, it is necessary to prove both the Theorem A. 1 and Theorem A. 2 which were used without proof. However, as will be seen later, the Theorem A.2 will be proved by slightly modifying the proof of the following theorem:

Theorem A. 3. Under the same assumptions as in Theorem A, the equation (A) admits a solution $y=S(x, U)$, where the n-vector 
$S(x, u)$ is a function holomorphic and bounded in $(x, u)$ for the domain (A.6) and admits the asymptotic expansion

$$
S(x, u) \simeq u+\mathbf{1}_{n}(u) \sum_{|\mathcal{K}|=1}^{\infty} P_{\mathcal{K}}^{(0)} u \mathcal{K}+\sum_{\substack{k, \mathcal{K} \\ k \geqq 1}} P_{\mathcal{K}}^{(k)} x^{k} u \mathcal{K}
$$

as $x$ and $u$ tend to 0 in the domain (A.6).

Remarks 1. The meaning of the asymptotic expansion ( $\mathrm{g}$ ) is as follows: For any positive integer $N>1$, we have the inequality

$$
\begin{gathered}
\left\|S(x, u)-u-\mathbf{1}_{n}(u) \sum_{|\mathcal{K}|=1}^{N-2} P_{\mathcal{K}}^{(0)} u \mathcal{K}-\sum_{k+|\mathcal{K}|=1}^{N-1} P_{\mathcal{K}}^{(k)} x^{k} u \mathcal{K}\right\| \\
\leqq K_{N}\left(|x|^{N}+\|u\|^{N}\right)
\end{gathered}
$$

for the domain (A.6), where $K_{N}$ is a certain positive constant.

2. The power series (g) coincides formally with the power series (f) if we replace the $n$-vectors $P^{(k)}(u)(k \geqq 0)$ by the power series $(\mathrm{A} .10)^{k}$.

$4^{\circ}$. Our purpose in the present note is to prove the Theorem A. 1, Theorem A. 2 and Theorem A. 3.

In Chapter I, we shall discuss about a formal tranformation and prove Theorem A.1. The Chapter II will be devoted to the investigation of the growth of a general solution $U\left(x, x_{0}, u^{0}\right)$ of the simplified equation $(\mathrm{R})$ near the origin $x=0$. We shall prove some fundamental inequalities (See Theorems 6.1 and 7.1) about the growth of the solution $U$. The Theorem A.3 will be proved in Chapter III. In the last Chapter, we shall give a brief sketch of the proof of Theorem A.2.

$5^{\circ}$. We shall explain the notation.

For the $n$-vector $y$, we denote its $j^{\text {th }}$ entry by $y_{j}$ and $\|y\|=\max _{j=1}^{n}\left|y_{j}\right|$ is the norm of $y$. The expression $\mathbb{1}_{n}(y)$ denotes an $n$-dimensional diagorial matrix such that the $n$-vector formed by the diagonal components coincides with the vector $y$.

$\mathbb{Z}_{n}$ is the $n$-by- $n$ unit-matrix.

$\boldsymbol{e}_{j}$ is the $n$-dimensional row unit-vector such that its $j^{\text {th }}$ component only is non-zero and equal to 1 . 
$\mathbf{1}$ is an $n$-dimensional column vector whose components are all equal to 1 .

The norm of the $n$-by- $n$ matrix $F$ is given by: $\|F\|=\max _{j} \sum_{k=1}^{n}\left|F_{j k}\right|$, $F_{j k}$ being $(j, k)$-entry of the matrix $F$.

For the $n$-vector $y$, the symbol $\langle y\rangle$ stands for the $n$-vector which is obtained by replacing every entry $y_{j}$ by its module.

For the $n$-vectors $y$ and $z$, the vectorial inequality $\langle y\rangle \leqq\langle z\rangle$ means that we have the inequality $\left|y_{j}\right| \leqq\left|z_{j}\right|$ for every $j$.

For the $n$-by- $n$ matrix $F$, let $F_{j k}$ be $(j, k)$-entry of the matrix $F$. The symbol $\langle F\rangle$ stands for the $n$-by- $n$ matrix formed by $\left\{\left|F_{j k}\right|\right\}$.

$\oplus$ means the direct sum.

To simplify the description, we use the following symbols for the scalar $w$ and the $n$-vector $\beta$ :

$$
\begin{aligned}
w^{\beta} & =\left(w^{\beta_{1}}, w^{\beta_{2}}, \cdots, w^{\beta_{n}}\right), \\
\arg \beta & =\left(\arg \beta_{1}, \arg \beta_{2}, \cdots, \arg \beta_{n}\right), \\
\operatorname{Re} \beta & =\left(\operatorname{Re} \beta_{1}, \operatorname{Re} \beta_{2}, \cdots, \operatorname{Re} \beta_{n}\right) \\
\|\beta\| & =\max _{j}\left|\beta_{j}\right|, \quad\|\beta\|^{\prime}=\min _{j}\left|\beta_{j}\right| .
\end{aligned}
$$

The components of the $n$-row vectors $\mathcal{K}=\left(k_{1}, k_{2}, \cdots, k_{n}\right)$ and $\mathscr{H}=\left(h_{1}, h_{2}, \cdots, h_{n}\right)$ are all nonnegative integers and $|\mathcal{K}|=k_{1}+k_{2}+\cdots+k_{n}$. The symbol $\mathcal{K}\{\mathscr{H}$ means that $|\mathcal{K}|<|\mathscr{H}|$ and we say that $\mathcal{K}$ precedes $\mathcal{H}$. The symbol $y^{\mathcal{K}}$ stands for the scalar expression

$$
y \mathcal{K}=y_{1}^{k_{1}} \mathcal{Y}_{2}^{k_{2}} \cdots y_{n^{n}}^{k_{n}} .
$$

For the $n$-dimensional columin vector function $f(y)$, the symbols $\frac{\partial}{\partial y} f(y)$ and $\frac{\partial \mathcal{K}}{\partial y \mathcal{K}} f(y)$ denote $n$-by-n matrices defined by the expressions:

$$
\begin{aligned}
\frac{\partial}{\partial y} f(y) & =\left(\frac{\partial}{\partial y_{1}} f(y), \frac{\partial}{\partial y_{2}} f(y), \cdots, \frac{\partial}{\partial y_{n}} f(y)\right), \\
\frac{\partial \mathcal{K}}{\partial y^{K}} f(y) & =\left(\frac{\partial^{k_{1}}}{\partial y_{1}^{k_{1}}} f(y), \frac{\partial^{k_{2}}}{\partial y_{2}^{k_{2}}} f(y), \cdots,-\frac{\partial^{k_{n}}}{\partial y_{n}^{k_{n}}} f(y)\right) .
\end{aligned}
$$

When $f(y)$ is an $n$-dimensional vector function developable in 
power series of $y$, the symbol $[f(y)]_{M}$ or, simply, $[y]_{M}$ means that its expansion lacks the terms of the degree less than $M$.

For the $n$-dimensional row vector $\mathcal{K}$ and the $n$-dimensional column vector $y$, the symbol $(\mathcal{K} \cdot y)$ means the inner product.

For the $n$-vector $y$, the symbol $y^{T}$ is its transposed vector.

For the $n$-by- $n$ matrix $F, F^{-1}$ is its inverse matrix.

Terminating the introduction, the author wishes to express his cordial thanks to Professor Wolfgang Wasow for his valuable advice.

\section{Chapter I. Formal transformation (Proof of Theorem A.1).}

\section{First step of formal transformation.}

The equation (A) is of the form

$$
x y^{\prime}=y \mathscr{M l} \mathbf{1}_{n}\left(f^{(0)}(y)\right) y+\sum_{k=1}^{\infty} f^{(k)}(y) x^{k} .
$$

1. 1. We shall first prove that the assumption

$$
f^{(1)}(0)=0
$$

does not harm any generality of our discussion.

Proof. If (1.2) is not true, we make a transformation of the form

$$
y=z+f^{(1)}(0) x
$$

Then, the transformed equation will be written as

$$
x z^{\prime}=z^{\mathscr{M}} \mathbb{1}_{n}\left(f^{(0)}(z)\right) z+\sum_{k=1}^{\infty} g^{(k)}(z) x^{k},
$$

where $g^{(k)}(z)$ are $n$-dimensional column vector functions with the same properties as the vector functions $f^{(k)}(y)$ and, especially,

$$
\begin{aligned}
& g^{(1)}(z)=f^{(1)}(z)+F(z) f^{(1)}(0)-f^{(1)}(0), \\
& F(z)=\frac{\partial}{\partial z}\left(z^{\Re / \mathbb{H}_{n}}\left(f^{(0)}(z)\right) z\right) .
\end{aligned}
$$

We shall now show that $F(0)=0$. Our assertion results immediately from this fact.

By a simple calculation we have 


$$
\begin{aligned}
F(z)= & \left(m_{1} z^{M-e_{1}} \mathbf{1}_{n}\left(f^{(0)}(z)\right) z, \cdots, m_{n} z^{M-e_{n}} \mathbf{1}_{n}\left(f^{(0)}(z)\right) z\right) \\
& +z^{M}\left(\frac{\partial}{\partial z_{1}} \mathbf{1}_{n}\left(f^{(0)}(z)\right) \cdot z, \cdots, \frac{\partial}{\partial z_{n}} \mathbf{1}_{n}\left(f^{(0)}(z)\right) \cdot z\right) \\
& +z^{M}\left(\mathbf{1}_{n}\left(f^{(0)}(z)\right) \frac{\partial}{\partial z_{1}} z, \cdots, \mathbf{1}_{n}\left(f^{(0)}(z)\right) \frac{\partial}{\partial z_{n}} z\right) \\
= & z^{M} \mathbf{1}_{n}\left(f^{(0)}(z)\right) z\left(m_{1} z^{-e_{1}}, \cdots, m_{n} z^{-e_{n}}\right) \\
& +z^{M}\left(\mathbf{1}_{n}(z) \frac{\partial}{\partial z_{1}} f^{(0)}(z), \cdots, \mathbf{1}_{n}(z) \frac{\partial}{\partial z_{n}} f^{(0)}(z)\right) \\
& +z^{M} \mathbf{1}_{n}\left(f^{(0)}(z)\right) .
\end{aligned}
$$

Hence, we have the identity

$$
\begin{aligned}
& F(z) \equiv \frac{\partial}{\partial z}\left(z^{\mathscr{M}} \mathbf{1}_{n}\left(f^{(0)}(z)\right) z\right) \\
&=z^{\mathscr{M}} \mathbf{1}_{n}\left(f^{(0)}(z)\right) z \mathscr{M} \mathbf{1}_{n}(z)^{-1}+z^{M} \mathbf{1}_{n}(z) \frac{\partial f^{(0)}(z)}{\partial z} \\
& \quad+z^{\mathscr{M}} \mathbf{1}_{n}\left(f^{(0)}(z)\right) .
\end{aligned}
$$

However, since $|\mathscr{M}| \geqq 1$, we see from this identity that $F(0)=0$, which proves our assertion.

Q.E.D.

1. 2. Next we make a transformation of the form

$$
y=z+p^{(N)}(z) x^{N}, \quad N \geqq 1,
$$

where $p^{(N)}(z)$ is an $n$-dimensional column vector whose components are formal power series of $z$.

Let

$$
x z^{\prime}=z^{M} \mathbf{1}_{n}\left(f^{(0)}(z)\right) z+\sum_{k=1}^{\infty} g^{(k)}(z) x^{k}
$$

be the equation derived from (1.1) by the formal transformation $(1.6)^{N}$. Then, we shall prove that:

We have the relation

$$
\begin{aligned}
& (1.8)^{N} \quad g^{(k)}(z)=f^{(k)}(z) \quad(k<N), \\
& (1.9)^{N} \quad g^{(N)}(z)=f^{(N)}(z)+F(z) p^{(N)}(z)-N p^{(N)}(z) \\
& -z^{\mathscr{M}} \frac{\partial p^{(N)}(z)}{\partial z} \mathbb{1}_{n}\left(f^{(0)}(z)\right) z
\end{aligned}
$$

where $F(z)$ is given by (1.4). 
Proof. The inverse transformation of $(1.6)^{N}$ can be written as $(1.10)^{N}$

$$
z=y-p^{(N)}(y) x^{N}+[x]_{2 N} .
$$

Differentiating the relation $(1.10)^{N}$ with respect to $x$, we have

$$
\begin{aligned}
x z^{\prime}=x y^{\prime} & -\left(N p^{(N)}(y)+\frac{\partial p^{(N)}(y)}{\partial y} x y^{\prime}\right) x^{N}+[x]_{2 N} \\
= & y \mathscr{M} \mathbf{1}_{n}\left(f^{(0)}(y)\right) y+\sum_{k=1}^{N} f^{(k)}(y) x^{k} \\
& \quad-\left(N p^{(N)}(y)+\frac{\partial p^{(N)}(y)}{\partial y} y \mathscr{M} \mathbf{1}_{n}\left(f^{(0)}(y)\right) y\right) x^{N}+[x]_{N+1} .
\end{aligned}
$$

Substituting $(1.6)^{N}$ for $y$, we can easily verify that the relations $(1.8)^{N}$ and $(1.9)^{N}$ are satisfied.

Q.E.D.

Now we consider the partial differential equation

$$
z^{\mathscr{M}} \frac{\partial p}{\partial z} \mathbf{1}_{n}\left(f^{(0)}(z)\right) z=-N p+F(z) p+f^{(N)}(z),
$$

which is obtained by putting $g^{(N)}(z) \equiv 0$ in $(1.9)^{N}$.

We can assert that the equation (1.11) admits a formal solution of the form

$$
p \sim \sum_{\mathcal{K}} p_{\mathcal{K}} z^{\mathcal{K}}
$$

where $p_{\mathcal{K}}$ are $n$-dimensional constant column rectors.

Proof. Noticing that

$$
\mathbb{1}_{n}\left(f^{(0)}(z)\right) z \equiv \mathbb{1}_{n}(z) f^{(0)}(z),
$$

we put the formal solution (1.12) into the equation (1.11). Then, we have

$$
\begin{aligned}
& \sum_{\mathcal{K}}\left(\mathcal{K} \cdot f^{(0)}(z)\right) p_{\mathcal{K}} z^{\mathcal{K}+\mathscr{K}} \\
=- & N \sum_{\mathcal{K}} p_{\mathcal{K}} z^{\mathcal{K}}+\sum_{\mathcal{K}} F(z) p_{\mathcal{K}} z^{\mathcal{K}}+f^{(N)}(z) .
\end{aligned}
$$

However, as we have just seen, $F(0)=0$ and $|\mathscr{M}| \geqq 1$. Therefore, we get the equation

$$
\begin{aligned}
& N p_{\mathcal{K}}=-\sum_{\mathscr{H}, \mathcal{L}}\left(\mathscr{H} \cdot f_{\mathcal{L}}^{(0)}\right) p_{\mathscr{H}}+\sum_{\mathscr{H}} F_{\mathscr{H}} p_{\mathcal{K}-\mathscr{H}}+f_{\mathcal{K}}^{(N)},
\end{aligned}
$$

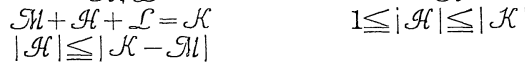


where $f_{\mathcal{K}}^{(0)}, f_{\mathcal{K}}^{(N)}$ and $F_{\mathcal{K}}$ stand for the coefficients of the terms $z^{\mathcal{K}}$ in the uniformly convergent expansions of the functions $f^{(0)}(z), f^{(N)}(z)$ and $F(z)$ in power series of $z$ respectively. From this recurrence formula we see that the coefficient vector $p_{\mathcal{K}}$ is expressed by a linear form of the coefficient vectors $p_{\mathscr{H}}$ such that $\mathcal{H}\{\mathcal{K}$. This proves the existence of the formal solution (1.12).

Q.E.D.

1.3. Thus, applying successively formal transformations of the form

$$
\left\{\begin{array}{c}
y=z_{1}+P^{(1)}\left(z_{1}\right) x \\
z_{1}=z_{2}+P^{(2)}\left(z_{2}\right) x^{2} \\
\cdots \cdots \cdots \cdots \cdots \cdots \cdots \\
z_{N-1}=z_{N}+P^{(N)}\left(z_{N}\right) x^{N} \\
\cdots \cdots \cdots \cdots \cdots \cdots
\end{array}\right.
$$

we have the following proposition:

Proposition 1.1. There exists a formal transformation of the form

$$
y \sim z+\sum_{k=1}^{\infty} p^{(k)}(z) x^{k}
$$

such that the equation (1.1) is formally transformed into the equation

$$
x z^{\prime}=z^{M \mathbb{1}_{n}}\left(f^{(0)}(z)\right) z=z^{M \mathbb{1}_{n}(z) f^{(0)}(z)}
$$

by the formal transformation (1.16). Here, the n-vectors $p^{(k)}(\approx)$ are formal power series of $z$ and the n-vector $f^{(0)}(z)$ is the same as in the equation (1.1).

\section{Second step of formal transformation.}

We try to transform the equation (1.17) into as simple an equation as possible.

The equation (1.17) can be written as

$$
x z^{\prime}=\approx \mathfrak{s}_{1} \mathbb{H}_{n}(z)\left(\alpha+\sum_{|\mathcal{K}| \geqq 1} \alpha_{\mathcal{K}} z^{J}\right) .
$$

2.1. Now we consider a transformation of the form

$$
z=u+\mathbb{1}_{n}(u) \sum_{|\varkappa|=N} p_{\mathcal{K}} u K
$$


where $p_{\mathcal{K}}$ are $n$-dimensional constant column vectors.

The inverse transformation of $(2.2)^{N}$ can be written as

$$
u=z-\mathbf{1}_{n}(z) \sum_{|\mathcal{K}|=N} p_{\mathcal{K}} z^{\mathcal{K}+\mathbf{1}_{n}(z)[z]_{2 N} .}
$$

Differentiating $(2.3)^{N}$ term by term, we have

$$
\begin{aligned}
x u^{\prime} & =x z^{\prime}-x\left(\mathbf{1}_{n}(z)\right)^{\prime} \sum_{\mathcal{K}} p_{\mathcal{K}} z^{\mathcal{K}} \quad\left({ }^{\prime}=\frac{d}{d x}\right) \\
& -\mathbf{1}_{n}(z) \sum_{\mathcal{K}} p_{\mathcal{K}} x\left(z^{\mathcal{K}}\right)^{\prime}+x\left(\mathbf{1}_{n}(z)[z]_{2 N}\right)^{\prime} .
\end{aligned}
$$

It is easily verified that

$$
\begin{aligned}
& x\left(\mathbf{1}_{n}(z)\right)^{\prime}=z^{\mathscr{M}} \mathbf{1}_{n}(z) \mathbf{1}_{n}\left(f^{(0)}(z)\right), \\
& x\left(z^{\mathcal{K}}\right)^{\prime}=z^{M}+\mathcal{K}\left(\mathcal{K} \cdot f^{(0)}(z)\right) \text {, } \\
& x\left(\mathbf{1}_{n}(z)[z]_{2 N}\right)^{\prime}=z^{M} \mathbf{1}_{n}(z)[z]_{2 N} .
\end{aligned}
$$

Hence, we have the equation

$$
\begin{aligned}
x u^{\prime} & =z^{\mathscr{M}} \mathbf{1}_{n}(z)\left(f^{(0)}(z)-\mathbb{1}_{n}\left(f^{(0)}(z)\right) \sum_{\mathcal{K} \mid=N} p_{\mathcal{K}} z^{\mathcal{K}}\right. \\
& \left.-\sum_{|\mathcal{K}|=N}\left(\mathcal{K} \cdot f^{(0)}(z)\right) p_{\mathcal{K}} z^{\mathcal{K}}+[z]_{2 N}\right) .
\end{aligned}
$$

From this we see that the transformed equation (i.e. the equation satisfied by $u$ ) has also the same form as (2.1):

$$
x u^{\prime}=u \mathscr{M} \mathbf{1}_{n}(u)\left(\alpha+\sum_{\mathcal{K}} \beta_{\mathcal{K}} u \mathcal{K}\right)
$$

and, especially,

$$
\beta_{\mathcal{K}}= \begin{cases}\alpha_{\mathcal{K}} & \text { if } \quad|\mathcal{K}|<N, \\ \alpha_{\mathcal{K}}-(\mathcal{K} \cdot \alpha) p_{\mathcal{K}}+\left(\mathscr{H} \cdot p_{\mathcal{K}}\right) \alpha & \text { if } \quad|\mathcal{K}|=N .\end{cases}
$$

The last relation in $(2.6)^{N}$ can be easily verified by picking up the terms of the form $u^{M} \mathbb{1}_{n}(u)[\cdots] u \mathcal{K}$ for $|\mathcal{K}|=N$, the symbol $[\cdots]$ standing for an $n$-dimensional column vector, in the expression $z^{\mathscr{M}} \mathbb{1}_{n}(z) f^{(0)}(z)$ with $z=\imath+\mathbb{1}_{n}(u) \sum_{\mathcal{K}} p_{\mathcal{K}} u \mathcal{K}$. These terms are given by

$$
\begin{aligned}
& u \mathscr{M} \mathbb{1}_{n}(u) \alpha_{\mathcal{K}} u \mathcal{K}+\frac{\partial}{\partial u}\left(u \mathscr{M} \mathbb{1}_{n}(u) \alpha\right) \mathbb{1}_{n}(u) p_{\mathcal{K}} u \mathcal{K} \\
= & u \mathscr{M} \mathbf{1}_{n}(u)\left(\alpha_{\mathcal{K}}+\left(\mathscr{M} \cdot p_{\mathcal{K}}\right) \alpha+\mathbf{1}_{n}(\alpha) p_{\mathcal{K}}\right) u \mathcal{K} .
\end{aligned}
$$


2.2. Now we consider the linear equation

$$
(\mathcal{K} \cdot \alpha) p-(\mathscr{H} \cdot p) \alpha=\alpha_{\mathcal{K}}-\beta_{\mathcal{K}} \quad\left(p=p_{\mathcal{K}}\right) .
$$

As can be easily seen, this equation can be written as

$$
L p=\alpha_{\mathcal{K}}-\beta_{\mathcal{K}},
$$

where

$$
L=(\mathcal{K} \cdot \alpha) \mathbf{1}_{n}-\alpha \mathscr{M} .
$$

We can prove that the determinant of the matrix $L$ is equal to $(\mathcal{K} \cdot \alpha)^{n-1}((\mathcal{K}-\mathscr{M}) \cdot \alpha)$, i.e.

$$
\operatorname{det} L=(\mathcal{K} \cdot \alpha)^{n-1}((\mathcal{K}-\mathscr{M}) \cdot \alpha) \text {. }
$$

The proof of this formula will be given in the next section.

Since the assumption $v$ ) in Introduction is supposed to be satisfied, the quantity $(\mathcal{K} \cdot \alpha)$ never vanishes if $|\mathcal{K}| \geqq 1$. Hence, the determinant of $L$ is equal to zero if and only if the vector $\mathcal{K}$ satisfies the equation

$$
((\mathcal{K}-\mathscr{M}) \cdot \alpha)=0 .
$$

We denote the set of vectors $\mathcal{K}$ for which we have $(2 \cdot 9)$ by $\mathfrak{S}$. Clearly, $\mathfrak{S}$ is a finite set.

Thus we have the following proposition:

If $\mathcal{K} \notin S$, we can determine the value of the coefficient vector $p_{\mathcal{K}}$ of the formal transformation $(2.2)^{N}$ so that the value of the $n$-vecotr $\beta_{\mathcal{K}}$ is zero.

2.3. Suppose $\mathcal{K} \in \mathfrak{S}$. Then, the determinant of the matrix $L$ vanishes. We can assert that the rank of the matrix $L$ with $(\mathcal{K} \cdot \alpha)$ $=(\mathscr{M} \cdot \alpha)$ is equal to $n-1$.

Proof. Since $|\mathscr{M}| \geqq 1$, there exists at least one index $j^{*}$ such that $m_{j} * \neq 0$. Then, we can prove that the determinant of the $(n-1)-b y$ $(n-1)$ matrix $L_{j}^{*}$ which is obtained by removing the components of the $j^{* \text { th }}$ row and the $j^{* \text { th }}$ column of the matrix $L$ is equal to $m_{j}^{*} \alpha_{j}^{*}(\mathscr{M} \cdot \alpha)^{n-2}$, i.e.

$$
\operatorname{det} L_{j}^{*}=m_{j}^{*} \alpha_{j}^{*}(\mathscr{M} \cdot \alpha)^{n-2}
$$


This determinant is non-zero and we have proved our assertion. Q.E.D. The proof of the equality (II) will be given in the next section.

Moreover, if $\mathcal{K} \in \mathfrak{S}$, as can be easily verified, we have

$$
\mathscr{M L}=0 .
$$

On the other hand, we can assume, without loss of generality, that the vector $\alpha$ is expressed by a direct sum of $\kappa$ column vectors $\alpha^{(i)}$ with dimension $n_{i}$ as follows:

$$
a^{\prime}=\alpha^{(1)} \oplus a^{(2)} \oplus \cdots \oplus \boldsymbol{\alpha}^{(k)}
$$

with the property that two components of the vector $\alpha$ are commensurable if and only if both of them are components of the same $\alpha^{(i)}$ for some $i$.

Then, there exist $\kappa$ non-zero complex constants $\gamma$. and $\kappa$ column vectors $1 i^{(i)} n_{i}$ with dimension $n_{i}$ such that

$$
\alpha^{(i)}=\gamma_{i} \mu^{(i)}
$$

and the components of the vectors $\mu^{(i)}$ are all positive integers. Moreover, the constant $\gamma_{i}$ is uniquely determined under the condition that the greatest common divisor of the components of the vector $\mu^{(i)}$ is equal to 1 or $\mu^{(i)}=1$ according as we have $n_{i}>1$ or $n_{i}=1$.

Hence, the equation (2.9) becomes equivalent to $\kappa$ equations:

$$
\left(\left(\mathcal{K}_{i}-\mathscr{M}_{i}\right) \cdot \alpha^{(i)}\right)=0, \quad(i=1,2, \cdots, \kappa),
$$

with

$$
\mathcal{K}=\mathcal{K}_{1} \oplus \mathcal{K}_{2} \oplus \cdots \oplus \mathcal{K}_{\kappa}, \quad \mathscr{M}=\mathscr{M}_{1} \oplus \mathscr{M}_{2} \oplus \cdots \oplus \mathscr{M}_{\kappa} .
$$

We divide the $n$-vector $\beta_{\mathcal{K}}$ into $\kappa$ vectors as well as the $n$-vector $\alpha$ as follows:

$$
\beta_{\mathcal{K}}=\beta_{\mathcal{K}}^{(1)} \oplus \beta_{\mathcal{K}}^{(2)} \oplus \cdots \oplus \beta_{\mathcal{K}}^{(\kappa)}, \quad \quad \beta_{\mathcal{K}}^{(i)}=\gamma_{i, \mathcal{K}} \mu^{(i)},
$$

where $\gamma_{i, \mathcal{K}}$ are certain complex constants.

Then, we can prove that we can determine the value of the coefficient vector $p_{\mathcal{K}}$ so that the n-vector $\beta_{\mathcal{K}}$ is of the form (2.12) if the complex constants $\gamma_{i, \mathcal{K}}$ are suitably chosen. 
Proof. It is enough to prove that we can choose the values of the constants $\gamma_{i, \mathcal{K}}$ so that the conditions

$$
\operatorname{rank} L=\operatorname{rank}\left(L, \alpha_{\mathcal{K}}-\beta_{\mathcal{K}}\right)=n-1
$$

are compatible. Since we have the relation (2.10), these conditions are equivalent to

$$
\left(\mathscr{M} \cdot\left(\alpha_{\mathcal{K}}-\beta_{\mathcal{K}}\right)\right)=0 .
$$

By the definition of the vector $\beta_{\mathcal{K}}$, this equation is written as

$$
\left(\mathscr{M} \cdot \alpha_{K}\right)-\sum_{i=1}^{\kappa} \gamma_{i, K}\left(M_{i} \cdot \mu^{(i)}\right)=0 \text {. }
$$

However, since at least one of the quantities $\left(\mathcal{M}_{i} \cdot \mu^{(i)}\right)$ is positive, the condition (2.13) can be certainily realized by a suitable choice of the constants $\gamma_{i, \mathcal{K}}$.

Q.E.D.

Thus, by applying successively formal transformations of the form

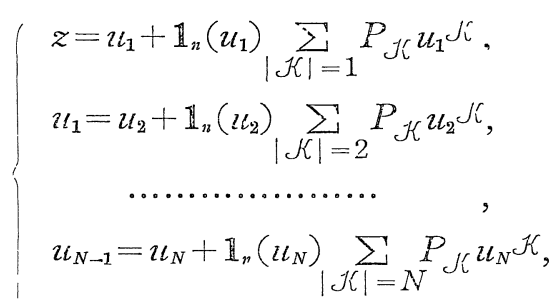

$\left(u_{j}(j=1,2, \cdots)\right.$ are $n$-dimensional column vectors) we have the following proposition:

Proposition 2.1. We can determine the values of the coefficient vectors $p_{\mathcal{K}}$ of a formal transformation of the form

$$
z \sim u+\mathbb{1}_{n}(u) \sum_{|\mathcal{K}| \geqq 1} p_{\mathcal{K}^{\prime}} u^{\mathcal{K}}
$$

so that the equation formally derived from the equation (2.1) by the formal transformation (2.15) takes the form

$$
\begin{aligned}
x u^{\prime} & =u^{\mathscr{M}} \mathbf{1}_{n}(u)\left(\alpha+\sum_{\mathscr{H} \in \mathbb{S}} \beta_{\mathscr{H}} u \mathscr{H}\right) \\
& =u^{\mathscr{M}}\left(\mathbf{1}_{n}(\alpha)+\sum_{\mathscr{H} \in \mathbb{S}} \mathbf{1}_{n}\left(\beta_{\mathscr{H}}\right) u \mathscr{H}\right) u .
\end{aligned}
$$


Here $\mathfrak{S}$ is the totality of the vectors $\mathscr{H}$ such that $((\mathscr{H}-\mathscr{M}) \cdot \alpha)=0$ and $\beta_{\mathscr{H}}$ are n-dimensional column constant vectors defined by the formula (2.12), and if $\beta_{\mathscr{G}} \neq 0$ we have

$$
\left((\mathcal{K}-\mathscr{M}) \cdot \beta_{\mathscr{H}}\right)=0 \quad \text { for any } \mathcal{K} \in S \text {. }
$$

Remark。 $\mathfrak{S}$ is a finite set, and the equation (2.16) is an immediate consequence of the equations $(2.9)_{i},(2.12)$ and (2.11).

\section{Proof of Theorem $\mathbb{A}$. 1 .}

A combined formal transformation of two formal transformations (1.16) and (2.15) can be obviously written in the form ( $f$ ) appearing in Theorem A. 1 in Introduction. By virtue of the Proposition 1.1 and Proposition 2.1, we have the Theorem A.1.

Therefore, for the complete proof of the Theorem A. 1, it remains only to prove the identities (I) and (II) appearing in Section 2:

$$
\begin{aligned}
& \operatorname{det} L=(\mathcal{K} \cdot \alpha)^{n-1}((\mathcal{K}-\mathscr{M}) \cdot \alpha) . \\
& \operatorname{det} L_{j} *=m_{j} * \alpha_{j} *(\mathscr{M} \cdot \alpha)^{n-2} .
\end{aligned}
$$

3. 1. We consider the following determinant:

$$
D(\mathscr{H}, \alpha)=\left|\begin{array}{ccccc}
b-m_{1} \alpha_{1} & -m_{2} \alpha_{1} & \cdots & \ldots & -m_{n} \alpha_{1} \\
-m_{1} \alpha_{2} & b-m_{2} \alpha_{2} & -m_{3} \alpha_{2} & \ldots & -m_{n} \alpha_{2} \\
\cdots & \cdots & \cdots & \ldots & \ldots \\
-m_{1} \alpha_{n} & \cdots & \cdots & -m_{n-1} \alpha_{n} & b-m_{n} \alpha_{n}
\end{array}\right| .
$$

Since $D(\mathscr{M}, \alpha)=\operatorname{det}\left(b \mathbf{1}_{n}-\alpha \mathscr{M}\right)$, it is obvious by (2.8) that

$$
\operatorname{det} L=D(\mathscr{M}, \alpha) \text { with } b=(\mathcal{K} \cdot \alpha) .
$$

We shall prove that

$$
D(\mathscr{M}, \alpha)=b^{n-1}(b-(\mathscr{M} \cdot \alpha)) .
$$

The formula (I) results immediately from (3.1).

Proof. Adding the components of ths $\approx 2^{\text {rd }}, \cdots, n^{\text {th }}$ columns respectively to the components of the $1^{\text {st }}$ column, we have 


$$
\begin{aligned}
& D(\mathscr{M}, \alpha)=\left|\begin{array}{ccccc}
b-|\mathscr{M}| \alpha_{1} & -m_{2} \alpha_{1} & \cdots & \cdots & -m_{n} \alpha_{1} \\
b-|\mathscr{M}| \alpha_{2} & b-m_{2} \alpha_{2} & \cdots & \cdots & -m_{n} \alpha_{2} \\
\ldots & \cdots & \ldots & \ldots & \ldots \\
b-|\mathscr{M}| \alpha_{n} & -m_{2} \alpha_{n} & \cdots & -m_{n-1} \alpha_{n} & b-m_{n} \alpha_{n}
\end{array}\right| \\
& \begin{array}{c}
=b\left|\begin{array}{ccccc}
1 & -m_{2} \alpha_{1} & \ldots & \ldots & -m_{n} \alpha_{1} \\
1 & b-m_{2} \alpha_{2} & -m_{3} \alpha_{2} & \ldots & -m_{n} \alpha_{2} \\
\ldots & \ldots & \ldots & \ldots & \ldots \\
1 & -m_{2} \alpha_{n} & \ldots & -m_{n-1} \alpha_{n} & b-m_{n} \alpha_{n}
\end{array}\right| \\
+(-1)|\mathcal{M}| \alpha_{1}\left|\begin{array}{ccccc}
1 & -m & \ldots & \ldots & -m m_{n} \\
\alpha_{2} & b-m_{2} \alpha_{2} & -m \tau_{3} \alpha_{2} & \ldots & -m_{n} \alpha_{2} \\
\ldots & \ldots & \ldots & \ldots & \ldots \\
\alpha_{n} & -m_{2} \alpha_{n} & \ldots & -m_{n-1} \alpha_{n} & b-m_{n} \alpha_{n}
\end{array}\right| .
\end{array}
\end{aligned}
$$

However, we see that the first determinant is equal to $b D\left(\mathscr{M}_{1}, \alpha^{1}\right)$, where

$$
\begin{aligned}
\left(\alpha^{1}\right)^{T} & =\left(\alpha_{2}^{1}, \cdots, \alpha_{n}^{1}\right) \quad \text { with } \quad \alpha_{j}^{1}=\alpha_{j}-\alpha_{1}, \\
\mathscr{M}_{1} & =\left(m_{2}, \cdots, m_{n}\right) .
\end{aligned}
$$

This can be easily verified by substructing the components of the $1^{\mathrm{s}}$ row from the components of every other row and by expanding the resulting determinant with respect to the components of the $1^{\text {ct }}$ column.

On the other hand, the last determinant can be easily calculated as follows: By adding the components of the $1^{\text {st }}$ row multiplied by $-\alpha_{j}$ to the components of the $j^{\text {th }}$ row for every $j$, the resulting determinant is reduced to a determinant of the upper triangular form and is equal to $(-1)|\mathcal{M}| \alpha_{1} b^{n-1}$.

Thus, we have the recurrence formula

$$
D\left(\mathscr{M}_{0}, \alpha^{0}\right)=b D\left(\mathscr{M}_{1}, \alpha^{1}\right)-\left|\mathscr{M}_{0}\right| \alpha_{1} b^{n-1},
$$

where

$$
\mathscr{M}_{0}=\mathscr{M}, \quad \alpha^{0}=\alpha
$$

Similarly, we can derive the following relations:

$$
D\left(\mathscr{H}_{1}, \alpha^{1}\right)=b D\left(\mathscr{M}_{2}, \alpha^{2}\right)-\left|\mathscr{M}_{1}\right| \alpha_{2}^{1} b^{n-2}
$$




$$
\begin{aligned}
& D\left(\mathscr{M}_{n-2}, \boldsymbol{\alpha}^{n-2}\right)=b D\left(\mathscr{M}_{n-1}, \boldsymbol{\alpha}^{n-1}\right)-\left|\mathscr{M}_{n-2}\right| \boldsymbol{\alpha}_{n-1}^{n-2} b \\
& D\left(\mathscr{M}_{n-1}, \boldsymbol{\alpha}^{n-1}\right)=b-\left|\mathscr{M}_{n-1}\right| \boldsymbol{\alpha}_{n}^{n-1}
\end{aligned}
$$

where

$$
\begin{aligned}
& \mathscr{M}_{k}=\left(m_{k+1}, \cdots, m_{n}\right), \\
& \left(\boldsymbol{\alpha}^{k}\right)^{T}=\left(\boldsymbol{\alpha}_{k+1}^{k}, \cdots, \boldsymbol{\alpha}_{n}^{k}\right) \quad \text { with } \quad \boldsymbol{\alpha}_{j}^{k}=\boldsymbol{\alpha}_{j}^{k-1}-\boldsymbol{\alpha}_{k}^{k-1} .
\end{aligned}
$$

Since, by definition of $\alpha_{j}^{k}$, we have

$$
\boldsymbol{\alpha}_{k}^{k-1}=\boldsymbol{\alpha}_{k}^{k-2}-\boldsymbol{\alpha}_{k-1}^{k-2}=\boldsymbol{\alpha}_{k}^{k-3}-\boldsymbol{\alpha}_{k-1}^{k-3}=\cdots=\boldsymbol{\alpha}_{k}-\boldsymbol{\alpha}_{k-1},
$$

it follows then that

$$
\begin{aligned}
D(\mathscr{M}, \alpha) & =b^{n}-\left(\left|\mathscr{M}_{0}\right| \alpha_{1}+\left|\mathscr{M}_{1}\right| \alpha_{2}^{1}+\cdots+\left|\mathscr{M}_{n-1}\right| \alpha_{n}^{n-1}\right) b^{n-1} \\
& =b^{n-1}(b-(\mathscr{H} \cdot \alpha)) .
\end{aligned}
$$

3.2. We can assume without loss of generality that $m_{1} \neq 0$. Since $(\mathcal{K} \cdot \alpha)=(\mathscr{H} \cdot a)$, the matrix $L_{1}$ is written as

$$
L_{1}=(\mathscr{M} \cdot \alpha) \mathbb{1}_{n-1}-\alpha^{*} \mathscr{M}_{1},
$$

where

$$
\mathscr{H}_{1}=\left(m m_{2}, \cdots, m_{n}\right), \quad\left(\alpha^{*}\right)^{T}=\left(\alpha_{2}, \cdots, \alpha_{n}\right) .
$$

This matrix has a form similar to the original matrix $L$ except that the size of the matrix $L_{1}$ is $n-1$ by $n-1$. Hence, we see that the determinant of $L_{1}$ is equal to $D\left(\mathscr{M}_{1}, \alpha^{*}\right)$ with $b=(\mathscr{M} \cdot \alpha)$. Therefore, the formula (3.1) shows us that

$$
\operatorname{det} L_{1}=(\mathscr{H} \cdot \alpha)^{n-2}\left((\mathscr{H} \cdot \alpha)-\left(\mathscr{M}_{1} \cdot \alpha^{*}\right)\right)=(\mathscr{M} \cdot \alpha)^{n-2} m_{1} \alpha_{1} \neq 0 .
$$

This proves the formula (II).

Chapter $\mathbb{I}$. Integration of the simplified equation (R)。

\section{Equations equivalent to the equation $(\mathbb{R})$.}

We introduce an auxiliary variable

$$
\widehat{w}=u^{M} \text {. }
$$

Then, by a direct calculation, we can derive from the equation (R) the following equations: 


$$
\left\{\begin{array}{l}
\widehat{\omega}^{2} \cdot \frac{d x}{\hat{a} \hat{w}}=x\left(\frac{1}{(\mathscr{M} \cdot \alpha)+\sum_{\mathscr{H} \in \mathbb{S}}\left(\mathscr{M} \cdot \beta_{\mathscr{H}}\right) u^{\mathscr{H}}}\right), \\
\widehat{\mathfrak{w}} \cdot \frac{d u}{d \hat{w}}=\mathbf{1}_{n}\left(\frac{\alpha+\sum \beta_{\mathscr{H}} \mathcal{U}^{\mathscr{H}}}{(\mathscr{M} \cdot \alpha)+\sum\left(\mathscr{M} \cdot \beta_{\mathscr{H}}\right) u^{\mathscr{H}}}\right) u .
\end{array}\right.
$$

Therefore, we see that the differential equation ( $\mathrm{R}$ ) is equivalent to a system of the equations (4.1) and (4.2).

On the other hand, we can prove the following proposition:

Proposition 4.1. Let $x(\widehat{\omega})$ and $u(\widehat{\omega})$ be the holomorphic solution of the equations (4.2) such that $x\left(\widehat{\omega}^{0}\right)=x_{0}$ and $u\left(\widehat{\omega}^{0}\right)=u^{0}$. Then, we have the identity $u(\hat{w}) \mathscr{M} \equiv w$ if and only if the relation $\left(u^{0}\right) M=\widehat{w}^{0}$ holds.

Proof. A simple calculation shows us that the expression $u(\widehat{\omega}) \mathscr{M}$ $-\widehat{w}$ is a solution of the linear differential $\widehat{w} \frac{d Y}{d \widehat{w}}=Y$. However, the solution of this equation is identically zero or never vanishes, which proves our proposition.

\section{Parametric representation of solution.}

5. 1. Let $U \equiv U\left(x, x_{0}, u^{0}\right)$ be the holomorhic solution of the equation (R) satisfying the initial condition $U=u^{0}$ at $x=x_{0}$.

Then, we can assert that:

Proposition 5.1. The function $U\left(x, x_{0}, u^{0}\right) \mathcal{J}-\mathscr{I l}$ is independent of $x$ for every $\mathcal{K} \in \mathfrak{S}$.

Proof. Since $U$ is a solution of the equation (R), by an elementary calculation we have

$$
x(U \mathcal{K}-\mathscr{M})^{\prime}=U \mathcal{K}\left\{((\mathcal{K}-\mathscr{M}) \cdot \alpha)+\sum_{\mathscr{H} \in \mathbb{S}}\left((\mathcal{K}-\mathscr{M}) \cdot \beta_{\mathscr{H}}\right) U^{\mathscr{H}}\right\} .
$$

By the definition of the set $\mathfrak{S}$, we have $((\mathcal{K}-\mathscr{M}) \cdot \alpha)=0$. On the other hand, the formula (2.12) implies that

$$
\left((\mathcal{K}-\mathscr{M}) \cdot \beta_{\mathscr{H}}\right)=\sum_{i=1}^{\kappa} \oplus \boldsymbol{r}_{i, \mathscr{H}}\left(\left(\mathcal{K}_{i}-\mathscr{M}_{i}\right) \cdot \mu^{(i)}\right)=0 .
$$

The last equality results immediately from the equation $(2.9)_{i}$ and the relation (2.11).

Q.E.D. 
Owing to this proposition, the $n$-vector $\alpha^{*}$ defined by

$$
\alpha^{*}=\sum_{\mathscr{H} \in \mathbb{S}^{\prime}} \beta_{\mathscr{H}} U\left(x, x_{0}, \iota^{0}\right) \mathscr{H}-\mathscr{M}
$$

does not depend on $x$. Therefore, we see, by virtue of the Proposition 4. 1 , that the solution $u=U\left(x, x_{0}, u^{0}\right)$ is equivalent to the solutions $x=\tilde{x}(w), u=\tilde{U}(w)$ of the equations

$$
\left\{\begin{array}{l}
w^{2} \frac{d x}{d w}=x\left(\frac{1}{(\mathcal{M} \cdot \alpha)+\left(\mathcal{M} \cdot \alpha^{*}\right) w}\right), \\
w \frac{d u}{d w}=\mathbb{1}_{n}\left(\frac{\alpha+\alpha^{*} w}{\left(\mathscr{M} \cdot \alpha^{\prime}\right)+\left(\mathcal{M} \cdot \alpha^{*}\right) w}\right) u
\end{array}\right.
$$

such that $\tilde{x}\left(w^{0}\right)=x_{0}$ and $\widetilde{U}\left(w^{0}\right)=u^{0}$ with

$$
w^{0}=\left(u^{0}\right) \mathscr{H}
$$

As we have already seen, the relation (5.2) implies that

$$
w \equiv \widetilde{U}(w) \mathscr{M}
$$

Since the coefficient matrix of the second equation of $\left(R^{\prime}\right)$ is of the diagonal form, we can integrate $\left(R^{\prime}\right)$ by quadratures as follows:

$$
\begin{aligned}
& \left\{\begin{array}{l}
\tilde{x}(w)=\hat{c}\left(1+\frac{(\mathscr{M} \cdot \alpha)}{\left(\mathscr{M} \cdot \alpha^{*}\right) w}\right)^{\frac{\left(\mathscr{M} \cdot \alpha^{*}\right)}{(\mathscr{N} \cdot \alpha)^{2}}} \exp \left(-\frac{1}{(\mathscr{M} \cdot \alpha) w}\right), \\
\tilde{U}(w)=\mathbb{1}_{n}\left(w^{\frac{\alpha}{(\mathscr{N} \cdot \alpha)}}\right) \mathbb{1}_{n}\left(\left((\mathscr{M} \cdot \alpha)+\left(\mathscr{M} \cdot \alpha^{*}\right) w\right)^{\left.\frac{\alpha^{*}}{\left(\mathscr{M} \cdot \alpha^{*}\right)}-\frac{\alpha}{(\mathscr{M} \cdot \alpha)}\right)} \hat{C}\right.
\end{array}\right. \\
& (\widehat{C} \mathscr{M}=1) \text {. }
\end{aligned}
$$

Here, the constant $\hat{c}$ and the $n$-dimensional column vector $\widehat{C}$ must be so chosen that $\tilde{x}\left(w^{0}\right)=x_{0}$ and $\tilde{U}\left(w^{0}\right)=u$.

5. 2. If we want to express $\widetilde{U}$ as a function of $x$, we must eliminate $w$ from the relations (5.4).

To do this, it is convenient to use a certain transcendental function introduced by M. Hukuhara [1, 4]. Following Hukuhara, we put

$$
x=c \exp \left(-\frac{\left(\mathscr{M} \cdot \alpha^{*}\right)}{(\mathscr{M} \cdot \alpha)^{2}} X\right), \quad w=\frac{(\mathscr{M} \cdot \alpha)}{\left(\mathscr{M} \cdot \alpha^{*}\right) W} .
$$


Then, the first equation of $\left(R^{\prime}\right)$ is transformed into

$$
\frac{d W}{d X}=1+\frac{1}{W} .
$$

By integrating this, we have

$$
X+\hat{c}_{1}=W-\log (W+1) .
$$

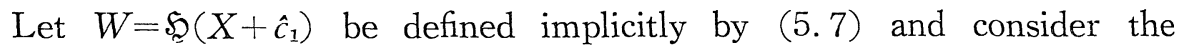
branch of $\mathfrak{S}(X)$ such that $\mathfrak{S}(X)-X-\log X$ vanishes at $X=\infty$. Then, the general solution $U$ of the equation $(R)$ can be written as

$$
\begin{gathered}
U=\mathbb{1}_{n}\left(\mathscr{S}\left(X+\hat{c}_{1}\right)-\frac{\alpha}{(\mathscr{M} \cdot \alpha)}\right) \mathbb{1}_{n}\left(\left((\mathscr{M} \cdot \alpha)\left(1+\mathscr{L}\left(X+\hat{c}_{1}\right)^{-1}\right)\right)^{\frac{\alpha^{*}}{\left(\mathscr{M} \cdot \alpha^{*}\right)}}-\frac{\alpha}{(\mathscr{M} \cdot \alpha)}\right) \widehat{C}, \\
\widehat{C} \mathscr{M}=1, \quad X=-\frac{(\mathscr{M} \cdot \alpha)^{2}}{\left(\mathscr{M} \cdot \alpha^{*}\right)} \log x .
\end{gathered}
$$

5. 3. Let

$$
\begin{aligned}
& D(c)=\left\{w: \theta_{-}+2 \delta_{0} \leqq \arg w \leqq \theta_{+}-2 \delta_{0}, 0<|w|<c\right\}, \\
& D^{*}(c)=\left\{w: \theta_{-}+2 \delta_{0}-\pi \leqq \arg w \leqq \theta_{+}-2 \delta_{0}+\pi, 0<|w|<c\right\},
\end{aligned}
$$

where

$$
\theta_{ \pm}=-\arg (\mathscr{M} \cdot \alpha) \pm \frac{\pi}{2},
$$

$\boldsymbol{\delta}_{0}$ being an arbitrarily fixed sufficiently small positive constant.

It should be noticed that, when $w$ tends to 0 along a segment in the domain $D(c)$, the function $\tilde{x}(w)$ defined by the first relation of (5.4) decreases monotonously in module and tends exponentially to 0 .

Then, the nature of the function $x=\tilde{x}(w)$ and of its inverse function is clarified by the following theorem:

Theorem 5.1. Let $x_{0}$ and $w^{0}$ be arbitrary points in the complex $x$-plane and in the domain $D^{*}(c)$ respectively. We choose a value of the constant $\hat{c}$ so that the first equation of (5.4) is satisfied at $x=x_{0}$ and $w=w^{\circ}$.

Then, if w tends to 0 along a curve $\Gamma_{w_{0}}^{*} \in D^{*}(c)$ (rohich starts 
from $w=w^{0}$ and approaches $w=0$ from the interior of the domain $D(c))$, the point $x=\tilde{x}(w)$ describes a spiral-shaped curve $\Gamma_{x_{0}}$, turning around the origin in the complex $x$-plane, and approaches $x=0$.

The converse is also true.

6. Estimation of the growth of the general solution of the equation ( $R$ ) near the origin $x=0$.

We consider a domain

$$
\mathfrak{D}\left(c_{0}\right)=\left\{w: \Theta_{1}<\arg w<\Theta_{2}, 0<|w|<d\left(\arg w, c_{0}\right)\right\},
$$

where $\Theta_{1}$ and $\Theta_{2}$ are the same as those appearing in (A. 8) in Introduction and

$$
d(\varphi, c)=c \exp \int_{\theta_{0}}^{\Phi} \cot A(\tau) d \tau, \quad\left(\Theta_{1} \leqq \theta_{0} \leqq \Theta_{2}\right)
$$

with

$$
A(\tau)= \begin{cases}\max \left(\tau-\theta_{+}+2 \delta_{0}, \delta_{0}\right), & \theta_{+}-2 \delta_{0} \leqq \tau \leqq \Theta_{2}, \\ \frac{\pi}{2}, & \theta_{-}+2 \delta_{0} \leqq \tau \leqq \theta_{+}-2 \delta_{0}, \\ \min \left(\tau-\theta_{-}+\pi-2 \delta_{0}, \pi-\delta_{0}\right), & \Theta_{1} \leqq \tau \leqq \theta_{-}+2 \delta_{0} .\end{cases}
$$

The point $c_{0} e^{i \theta_{0}}$ is evidently on the boundary of $\mathfrak{D}\left(c_{0}\right)$.

By the definition of $\theta_{-}, \theta_{+}, \Theta_{1}$ and $\Theta_{2}$, the function $A(\tau)$ satisfies the inequalities $\delta_{0} \leqq A(\tau) \leqq \pi-\delta_{0}$ for $\Theta_{1} \leqq \tau \leqq \Theta_{2}$. Hence, the function $d(\varphi, c)$ is bounded with respect to both variables. Therefore, the domain $\mathfrak{D}\left(c_{0}\right)$ is contained in the domain $\widetilde{\mathfrak{D}}\left(c^{\prime \prime}\right)$ defined by (A. 7) in Introduction for the suitably chosen positive constants $c$ and $c^{\prime \prime}$. The converse is also true.

By virtue of the assumption v) in Introduction, we can assume, without loss of generality, that

$$
\left\|\arg \frac{\boldsymbol{\alpha}}{(\mathscr{M} \cdot \boldsymbol{\alpha})}\right\| \leqq \frac{\pi}{2}-3 \delta_{0}
$$

for the same $\delta_{0}$ as before. Then, we can assert that we have

$$
D\left(c_{1}\right) \subset \mathfrak{D}\left(c_{0}\right) \subset D^{*}\left(c_{2}\right), \quad\left(c_{1}<c_{0}<c_{2}\right),
$$

where the domains $D(c)$ and $D^{*}(c)$ are defined by (5.9) and (5.10). 
On the study of a singular point of Briot-Bouquet type 329

Proof. We see by definition of $\theta_{-}, \theta_{+}, \Theta_{1}$ and $\Theta_{2}$ that

$$
\begin{aligned}
& \left(\theta_{+}+\pi-2 \delta_{0}\right)-\Theta_{2}=\frac{\pi}{2}+\max \arg \frac{\alpha}{(\mathcal{M} \cdot \alpha)}+2 \delta_{0} \geqq 5 \delta_{0}, \\
& \Theta_{2}-\left(\theta_{+}-2 \delta_{0}\right)=\frac{\pi}{2}-\max \arg \frac{\alpha}{(\mathcal{M} \cdot \alpha)}-2 \delta_{0} \geqq \delta_{0},
\end{aligned}
$$

i.e.

$$
\theta_{+}-2 \delta_{0}<\Theta_{2}<\theta_{+}+\pi-2 \delta_{0}
$$

and similarly that: $\theta_{-}-\pi+2 \delta_{0}<\Theta_{1}<\theta_{-}+2 \delta_{0}$.

Q.E.D.

Let $\left(x_{0}, u^{0}\right)$ be an arbitrary point in the domain

$$
|x|<a_{0}, \quad\left\|\imath_{i}\right\|<b_{0}, \quad u \mathscr{S} \in \mathfrak{D}\left(c_{0}\right)
$$

and define the function $U\left(x, x_{0}, u^{0}\right)$ as a solution of the equation (R) such that $U=u^{0}$ at $x=x_{0}$. The solution $u=U\left(x, x_{0}, u^{0}\right)$ is equivalent to the solutions $x=\tilde{x}(w), u=\tilde{U}(w)$ of the equations ( $\left.\mathrm{R}^{\prime}\right)$ such that $\tilde{x}\left(w^{0}\right)=x_{0}, \tilde{U}\left(w^{0}\right)=u^{0}$ with $w^{0}=\left(u^{0}\right) \mathscr{M}$. These solutions are expressed by the formula (5.4). Thus, we have the identities

$$
\widetilde{U}(w) \mathscr{M} \equiv w, \quad \widetilde{U}\left(\tilde{x}^{-1}(x)\right) \equiv U\left(x, x_{0}, u^{0}\right) .
$$

Moreover, we can prove the following theorem:

Theorem 6.1. There exists, in the domain $\mathfrak{D}\left(c_{0}\right)$, a curce $\Gamma_{w 0}^{*}$, which starts from $w=w^{0}$ and approaches $w=0$ along a segment in the sector $\theta_{-}+2 \delta_{0} \leqq \arg w \leqq \theta_{+}-2 \delta_{0}$, such that we have the inequalities

$$
\begin{aligned}
& \frac{d \mid \tilde{x}(w) !}{d s} \geqq \frac{\sin \delta_{0}}{2|(\mathscr{M} \cdot \alpha)||w|^{2}}|\tilde{x}(w)|, \\
& \frac{d\|\tilde{U}(w)\|}{d s} \geqq \frac{\|\alpha\|^{\prime} \sin \delta_{0}}{2|(\mathscr{M} \cdot \alpha)||w|}\|\tilde{U}(w)\|, \\
& \left|\frac{d w}{d s}\right|=1
\end{aligned}
$$

on the path $\Gamma_{w 0}^{*}$, where $s$ is the length of the curve $\Gamma_{w 0}^{*}$ measured from the origin to the point w.

This theorem shows us that, as $w$ tends to 0 from $w=w^{0}$, both $|\tilde{x}(w)|$ and $\|\tilde{U}(w)\|$ decrease monotonously. Hence, we have the inequalities 


$$
|\tilde{x}(w)| \leqq\left|x_{0}\right|,\|\tilde{U}(w)\| \leqq\left\|u^{0}\right\| \quad \text { for } \quad w \in \Gamma_{w 0}^{*} .
$$

Therefore, it follows from (6.5) that

$$
|\tilde{x}(w)|<a_{0},\|\tilde{U}(w)\|<b_{0}, \tilde{U}(w) \mathscr{M} \in \mathscr{D}\left(c_{0}\right) \quad \text { for } \quad w \in \Gamma_{w 0}^{*} .
$$

Clearly, the path $\Gamma_{w 0}^{*}$ belongs to the class of paths that appeared in Theorem 5.1. Let $\Gamma_{x_{0}}$ be a curve in the complex x-plane obtained by mapping the curve $\Gamma_{w 0}^{*}$ by the first relation of (5.4), where the constant $\hat{c}$ should be determined so that $w=w^{0}$ is mapped into $x=x_{0}$. Then, we see that $\Gamma_{x_{0}}$ is a spiral-shaped path which starts from $x=x_{0}$ and approaches $x=0$, turning around the origin in the complex $x$-plane.

By virtue of the inequalities (6.9) and the identities (6.5), we have the following theorem:

Theorem 6.2. When $x$ moves on the path $\Gamma_{x_{0}}$, the values of $x$ and $U\left(x, x_{0}, u^{0}\right)$ always remain in the domain (6.4).

Proof. It is sufficient to observe that the correspondence between the points on these two curves $\Gamma_{u 0}^{*}$ and $\Gamma_{x_{0}}$ is one to one.

Remark. The domain of the point $u$ satisfying the inequalities (6.4) is in the product space of the Riemann surfaces of $\log u$ $=\left(\log u_{1}, \cdots, \log u_{n}\right)$.

\section{Proof of Theorem 6.1 .}

7.1. Let $(r, \theta)$ and $(\rho, \varphi)$ be the polar coordinates of the point $w^{0}$ and the variable point $w$ on the path $\Gamma_{w 0}^{*}$ and let $A(\tau)$ be the same as before.

The curve $\Gamma_{w 0}^{*}$ is defined as follows:

If $\theta_{+}-2 \delta_{0} \leqq \theta<\Theta_{3}$, the path $\Gamma_{w 0}^{*}$ consists of a curvilinear part $\Gamma^{\prime}$ :

$$
\rho=r \exp \int_{\theta}^{\rho} \cot A(\tau) d \tau, \quad \theta_{+}-2 \delta_{0} \leqq \varphi \leqq \theta
$$

and of a rectilinear part $\Gamma^{\prime \prime}$ :

$$
0 \leqq \rho \leqq r \exp \int_{\theta}^{\theta_{-}-2 \varepsilon_{0}} \cot A(\tau) d \tau, \quad \varphi=\theta_{+}-2 \delta_{0} .
$$


On the study of a singular point of Briot-Bouquet type 331

If $\theta_{-}+2 \delta_{0} \leqq \theta \leqq \theta_{+}-2 \delta_{0}$, the path $\Gamma_{w 0}^{*}$ consists of only a rectilinear part $\Gamma^{\prime \prime}$ :

$$
0 \leqq \rho \leqq r, \quad \varphi=\theta
$$

If $\Theta_{1}<\theta \leqq \theta_{-}+2 \delta_{0}$, the path $\Gamma_{w 0}^{*}$ consists of a curvilinear part $\Gamma^{\prime}$ :

$$
\rho=r \exp \int_{\theta}^{\rho} \cot A(\tau) d \tau, \quad \theta \leqq \varphi \leqq \theta_{-}+2 \delta_{0}
$$

and of a rectilinear part $\Gamma^{\prime \prime}$ :

$$
0 \leqq \rho \leqq r \exp \int_{\theta}^{\theta_{-}+2 \delta_{0}} \cot A(\tau) d \tau, \quad \varphi=\theta_{-}+2 \delta_{0} .
$$

It is clear that the path $\Gamma_{w 0}^{*}$ thus defined is contained in the domain $\mathfrak{D}\left(c_{0}\right)$.

After a simple calculation we have, by $\left(R^{\prime}\right)$,

$$
\begin{aligned}
& \frac{1}{|x|} \frac{d|x|}{d s}=\operatorname{Re}\left(\frac{1}{\left.(\mathcal{M} \cdot X(w, U)) w^{2}\right)} \frac{d w}{d s}\right), \\
& \frac{1}{\|U\|} \frac{d\|U\|}{d s} \geqq \min _{j} \operatorname{Re}\left(\frac{X_{j}(w, U)}{(\mathscr{M} \cdot X(w, U))} \frac{1}{w} \frac{d w}{d s}\right),
\end{aligned}
$$

where

$$
U=\tilde{U}(\mathfrak{w}), x=\tilde{x}(w), X(w, u)=\alpha+\sum_{\mathscr{H} \in \mathbb{S}-\{\mathscr{M}\}} \beta_{\mathscr{H}} \mathfrak{u} \mathscr{H}+\beta_{\mathscr{M}} w,
$$

$X_{j}(w, u)$ being the $j^{\text {h }}$ component of the $n$-vector $X(w, u)$.

7.2. The rectilinear part $\Gamma^{\prime \prime}$. Since $s=\rho=|w|$ on this part, we get $d w / d s=e^{i \varphi}$. Hence, we have the equality (6.8), and the relations (7.3) and (7.4) are reduced to

$$
(7.3)^{\prime \prime} \quad \frac{1}{|x|} \frac{d|x|}{d s} \geqq \frac{1}{\rho^{2}} \operatorname{Re}\left(\frac{e^{-i \phi}}{(\mathscr{M} \cdot \alpha)}\left(1+\frac{(\mathscr{M} \cdot Y(w, U))}{(\mathscr{M} \cdot \alpha)}\right)^{-1}\right)
$$

and

$$
\begin{aligned}
(7.4)^{\prime \prime} \frac{1}{\|U\|} \frac{d\|U\|}{d s} \geqq \frac{1}{\rho} \min _{j} \operatorname{Re}\left(\frac{\alpha_{j}}{(\mathscr{M} \cdot \alpha)}(\right. & \left.1+\frac{Y,(w, U)}{\alpha_{j}}\right) \\
& \left.\times\left(1+\frac{(\mathscr{M} \cdot Y(w, U))}{(\mathscr{M} \cdot \alpha)}\right)^{-1}\right)
\end{aligned}
$$

respectively, where

$$
Y(w, u) \equiv X(w, u)-\alpha=\sum^{*} \beta_{\mathscr{H}} \mathfrak{u} \mathscr{I}+\beta_{\mathfrak{I}} w \cdot
$$

On the other hand, we observe that 
i) By definition of $\theta_{-}$and $\theta_{+}$, the inequality $\theta_{-}+2 \delta_{0} \leqq \varphi \leqq \theta_{+}-2 \delta_{0}$ implies that $|\varphi+\arg (\mathscr{N} \cdot \alpha)| \leqq \frac{\pi}{2}-2 \delta_{0}$.

ii) Since $|w|$ and $\|U\|$ are assumed to be sufficiently small, we can suppose without loss of generality that

(7. 6) $\left|\left(1+\frac{Y_{j}(w, U)}{\alpha_{j}}\right)\left(1+\frac{(\mathscr{M} \cdot Y(w, U))}{(\mathscr{M} \cdot \alpha)}\right)^{-1}\right|>\frac{1}{2}$,

(7. 7) $\left|\arg \left(1+\frac{Y_{j}(w, U)}{\alpha_{j}}\right)-\arg \left(1+\frac{(\mathscr{H} \cdot Y(w, U))}{(\mathscr{M} \cdot \alpha)}\right)\right|<\delta_{0}$.

Therefore, we can derive from (7.3)" and (7.4)" the following inequalities:

$$
\frac{1}{|x|} \frac{d|x|}{d s} \geqq \frac{\sin \delta_{0}}{2|(\mathscr{M} \cdot \alpha)| \rho^{2}}, \quad \frac{1}{\|U\|} \frac{d\|U\|}{d s} \geqq \frac{\|\alpha\|^{\prime} \sin \delta_{0}}{2 \mid(\mathscr{M} \cdot \alpha \mid) \rho},
$$

which proves the inequalities (6.6) and (6.7).

7. 3. The curvilinear part $\Gamma^{\prime}$. Here, $\rho$ is a function of $\varphi$ given by (7.1) or (7.2) according as we have $\theta_{+}-2 \delta_{0}<\theta$ or $\theta<\theta_{-}+2 \delta_{0}$. Hence, if we notice that the function $A(\tau)$ defined by (6.2) satisfies the inequality $\delta_{0} \leqq A(\tau) \leqq \pi-\delta_{0}$ for $\Theta_{1} \leqq \tau \leqq \Theta_{2}$, we have

$$
\frac{d w}{d \varphi}=r(\cot A(\varphi)+i), \quad i=\sqrt{-1}
$$

and

$$
d s= \pm \frac{\rho}{\sin A(\varphi)} d \varphi
$$

according as we have $\theta_{+}-2 \delta_{0} \leqq \varphi \leqq \theta$ or $\theta \leqq \varphi \leqq \theta_{-}+2 \delta_{0}$. Therefore,

$$
\frac{d w}{d s}= \pm e^{(A(\varphi)+\varphi) i}, \quad i=\sqrt{-1}
$$

which proves the equality (6.8).

To prove the inequalities (6.6) and (6.7), we consider first the following case:

I. Case when $\theta_{+}-2 \delta_{0}<\theta$. 
The inequalities (7.3) and (7.4) imply respectively that

(7. 3)' $\frac{1}{|x|} \frac{d|x|}{d s} \geqq \frac{1}{\rho^{2}} \operatorname{Re}\left(\frac{e^{(A(\mathcal{P})-\mathscr{\rho}) i}}{(\mathscr{M} \cdot \boldsymbol{\alpha})}\left(1+\frac{(\mathscr{M} \cdot Y(w, U))}{(\mathscr{M} \cdot \boldsymbol{\alpha})}\right)^{-1}\right)$

and

$$
\text { (7. 4)' } \begin{aligned}
\frac{1}{\|U\|} \frac{d\|U\|}{d s} \geqq \frac{1}{\rho} \min _{j} \operatorname{Re}\left(\frac{\alpha_{j} e^{A(\mathscr{P}) i}}{(\mathscr{M} \cdot \boldsymbol{\alpha})}\right. & \left(1+\frac{Y_{j}(w, U)}{\alpha_{j}}\right) \\
\times & \left.\left(1+\frac{(\mathscr{M} \cdot Y(w, U))}{(\mathscr{M} \cdot \boldsymbol{\alpha})}\right)^{-1}\right) .
\end{aligned}
$$

However, by means of the inequalities (7.5), (7.6) and (7.7), it is sufficient, for the proof of the inequalities (6.6) and (6.7), to show that, for the function $A(\varphi)$ already defined by (6.2), the inequalities

$$
\begin{gathered}
\left|A(\varphi)-\varphi-\arg (\mathscr{M} \cdot \alpha) \pm \delta_{0}\right| \leqq \frac{\pi}{2}-\delta_{0}, \\
\left|A(\varphi)+\arg \frac{\alpha_{j}}{(\mathcal{M} \cdot \alpha)} \pm \delta_{0}\right| \leqq \frac{\pi}{2}-\delta_{0} \quad(j=1, \cdots, n)
\end{gathered}
$$

are simultaneously satisfied for $\theta_{+}-2 \delta_{0} \leqq \varphi \leqq \Theta_{2}$.

By definition of $A(\varphi)$ we notice that

$$
A(\varphi)=\left\{\begin{array}{cr}
\varphi-\theta_{+}+2 \delta_{0}, & \text { for } \theta_{+}-\delta_{0} \leqq \varphi \leqq \Theta_{2}, \\
\delta_{0}, & \theta_{+}-2 \delta_{0} \leqq \varphi \leqq \theta_{+}-\delta_{0} .
\end{array}\right.
$$

Substituting $\varphi-\theta_{+}+2 \delta_{0}$ for $A(\varphi)$ in (7.8) and (7.9), we have

$$
\begin{aligned}
& \left|-\theta_{+}+2 \delta_{0}-\arg (\mathscr{M} \cdot \alpha) \pm \delta_{0}\right| \leqq \frac{\pi}{2}-\delta_{0} . \\
& \left|\varphi-\theta_{+}+2 \delta_{0}+\arg \frac{\alpha_{j}}{(\mathscr{M} \cdot \alpha)} \pm \delta_{0}\right| \leqq \frac{\pi}{2}-\delta_{0} .
\end{aligned}
$$

By the formula (5.11), these inequalities are written as

$$
\begin{aligned}
& \left|-\frac{\pi}{2}+2 \delta_{0} \pm \delta_{0}\right| \leqq \frac{\pi}{2}-\delta_{0}, \\
& \left|\varphi-\frac{\pi}{2}+\arg \alpha_{j}+2 \delta_{0} \pm \delta_{0}\right| \leqq \frac{\pi}{2}-\delta_{0}
\end{aligned}
$$

respectively.

We see at once that the first inequality is automatically satisfied. 
On the other hand, we can easily verify that the second inequality is satisfied in the interval

$$
-\min (\arg \alpha) \leqq \varphi \leqq \pi-\max (\arg \alpha)-4 \delta_{0}\left(\equiv \Theta_{2}\right)
$$

By virtue of (5.11) and (6.3), we have the inequality

$$
-\min (\arg \alpha) \leqq \frac{\pi}{2}-\arg (\mathscr{M} \cdot \alpha)-3 \delta_{0} \leqq \theta_{+}-3 \delta_{0}
$$

From this we see that the interval (7.10) contains the interval $\theta_{+}-\delta_{0} \leqq \varphi \leqq \Theta_{2}$.

Thus we have proved that the inequalities (6.6) and (6.7) are simultaneously satisfied in the interval $\theta_{+}-\delta_{0} \leqq \varphi \leqq \Theta_{2}$.

Substituting $\delta_{0}$ for $A(\varphi)$ in the inequalities (7.8) and (7.9), they are reduced to

$$
\begin{aligned}
& \left|\delta_{0}-\varphi-\arg (\mathcal{H} \cdot \alpha) \pm \delta_{0}\right| \leqq \frac{\pi}{2}-\delta_{0} \\
& \left|\delta_{0}+\arg \frac{\alpha_{j}}{(\mathcal{M} \cdot \alpha)} \pm \delta_{0}\right| \leqq \frac{\pi}{2}-\delta_{0} .
\end{aligned}
$$

The second inequality is automatically satisfed, because of the inequality (6.3). The first inequality is satisfied in the interval

$$
-\frac{\pi}{2}-\arg (\mathscr{M} \cdot \alpha)+3 \delta_{0} \leqq \varphi \leqq \frac{\pi}{2}-\arg \left(\mathscr{M} \cdot c_{i}\right)-\delta_{0}
$$

By definition of $\theta_{-}$and $\theta_{+}$, the first and the last expressions in the above inequality can be written as $\theta_{-}+3 \delta_{0}$ and $\theta_{+}-\delta_{0}$.

Thus we have proved that the inequalities (6.6) and (6.7) are simultaneously satisfied in the interval $\theta_{+}-2 \delta_{0} \leqq \varphi \leqq \theta_{+}-\delta_{0}$.

This completes the proof for the case when $\theta_{+}-2 \delta_{0} \leqq \theta$.

II. The proof for the case when $\theta \leqq \theta_{-}+2 \delta_{0}$ can be carried out in quite a similar way.

Q.E.D.

7.4. As can be easily seen from the proof of Theorem 6.1, we have at once the following theorem:

Wheorem \%.1. Let $\widetilde{U}_{j}(w)$ be the $j^{\text {th }}$ component of the n-vector function $\tilde{U}(w)$. Then, on the path $\Gamma_{u 0}^{*}$ we have the inequality 


$$
\frac{d\left|\tilde{U}_{j}(w)\right|}{d s} \geqq \frac{\|\alpha\|{ }^{\prime} \sin \delta_{0}}{2|(\mathcal{H} \cdot \alpha)||w|}\left|\tilde{U}_{j}(w)\right|
$$

which, in vectorial form, can be written

$$
\frac{d\langle\widetilde{U}(w)\rangle}{d s} \geqq \frac{\|\alpha\| \|^{\prime} \sin \delta_{0}}{2|(\mathscr{M} \cdot \alpha)||w|}\langle\widetilde{U}(w)\rangle .
$$

Proof. Since the coefficient matrix appearing in the equation ( $R$ ) in Section 5, is of the diagonal form. The proof of this theorem is exactly the same as that of Theorem 6.1.

\section{Chapter III. Main Theorem (Proof of Theorem A.3).}

\section{Preliminaries.}

8. 1. In Chapter $I$, we have proved that there exists the formal transformation $(f)$ :

$$
y \sim u+\mathbf{1}_{n}(u) P^{(0)}(u)+\sum_{k=1}^{\infty} P^{(k)}(u) x^{k}
$$

by which the equation (A) with (A.2) (i.e. the equation (1.1)) is formally transformed into the equation $(\mathrm{R})$ :

$$
x u^{\prime}=u \mathscr{N} \mathbf{1}_{n}\left(\alpha+\sum_{\mathscr{H} \in \mathbb{S}^{\prime}} \beta_{\mathcal{H}} u \mathscr{H}\right) u .
$$

Here, the $n$-vectors $P^{(k)}(u)(k \geqq 0)$ are formal power series of $u$ :

$$
(\text { A. } 10)^{k} \quad P^{(k)}(u) \sim \sum_{\mathcal{K}} P_{\mathcal{K}}^{(k)} u^{\mathcal{K}} .
$$

The formal transformation ( $f$ ) can be obtained by combining the two formal transformations (1.16) (in Proposition 1.1) and (2.15) (in Proposition 2.1). Therefore, we see at once that the power series $u+\mathbf{1}_{n}(u) P^{(0)}(u)$ with (A.10) $)^{0}$ coincides formally with the power series $u+\mathbf{1}_{n}(u) \sum_{\mathcal{K}} p_{\mathfrak{l}} u^{\mathcal{K}}$ appearing in (2.15). Moreoveover, after applying the formal transformation (1.16) to the equation (1.1), the expression $y \boldsymbol{g l} \mathbf{1}_{n}\left(f^{(0)}(y)\right) y$, which is the term independent of $x$ in the right-hand member of (1.1), rests invariable (See the equation (1.17) in Proposition 1.1). Therefore, the power series

$$
v \sim U+\mathbf{1}_{n}(U) \sum_{\mathcal{K} \mid \geqq 1} P^{(0)} U \mathcal{K}
$$


is a formal solution of the equation

$$
x v^{\prime}=v \mathscr{M} \mathbb{1}_{n}\left(f^{(0)}(v)\right) v,
$$

provided that $U$ is a solution of the equation (R).

From this it follows readily that we have the vectorial inequality

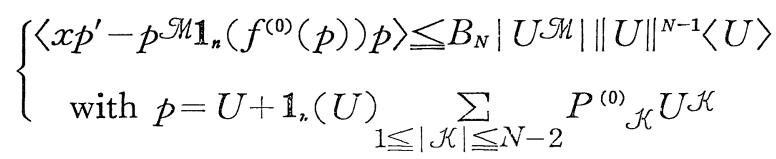

for $\|U\|<b^{\prime \prime}$, where $b^{\prime \prime}$ is a small positive number and $B_{N}$ is a certain positive constant.

8. 2. Let $F(p)$ be the $n$-by-n matrix defined by the formula (1.4) with $z=p$. Then, by (1.5) we have the vectorial equality

$$
\begin{aligned}
& \langle F(p)\rangle \equiv\left|p^{\mathscr{M}}\right| \mathbb{1}_{n}(\langle p\rangle)\langle\widehat{F}(p)\rangle \mathbb{1}_{n}(\langle p\rangle)^{-1}, \\
& \widehat{F}(p)=f^{(0)}(p) \mathscr{M}+f_{p}^{(1)}(p) \mathbb{1}_{n}(p)+\mathbb{1}_{n}\left(f^{(0)}(p)\right) .
\end{aligned}
$$

where $\widehat{F}(p)$ is an $n$-by-n matrix function holomorphic and bounded in $p$ for $\|p\|<b$. From this we see that there exists a positive constant $A_{1}$ such that

$$
\|F(p)\| \leqq A_{1}\|p\| \mid \Omega i
$$

and

$$
\langle F(p)\rangle\langle p\rangle \leqq A_{1}\left|p^{M L}\right|\langle p\rangle,
$$

because of the identity $\mathbb{1}_{n}(\langle p\rangle)^{-1}\langle p\rangle=\mathbb{1}$.

8. 3. Let $x_{0}$ and $u^{0}$ be arbitrary values such that

$$
\left|x_{0}\right|<a_{N}^{\prime \prime}, \quad\left\|u^{0}\right\|<b_{N}^{\prime \prime}, \quad\left(u^{0}\right) \mathscr{M} \in \mathfrak{D}\left(c_{N}^{\prime \prime}\right),
$$

where the positive constants $a_{N}^{\prime \prime}, b_{N}^{\prime \prime}$ and $c_{N}^{\prime \prime}$ will be defined later and the domain $\mathfrak{D}\left(c_{N}^{\prime \prime}\right)$ is defined by (6.1) with $c_{0}=c_{N}^{\prime \prime}$. We define the function $U\left(x, x_{0}, u^{0}\right)$ as the holomorphic solution of the equation (R) such that $U=u^{0}$ at $x=x_{0}$. Then, we have a formal solution of the form

$$
y \sim U+\mathbb{1}_{n}(U) \sum_{\mathcal{K}} P_{\mathcal{K}}^{(0)} U \mathcal{K}+\sum_{k, \mathcal{K}} P_{\mathcal{K}}^{(k)} x^{k} U \mathcal{K}
$$




\section{Auxiliary theorem.}

9. 1. Put

$$
P^{N}(x, u) \equiv u+\mathbf{1}_{n}(u) \sum_{|\mathcal{K}|=1}^{N-2} P_{\mathcal{K}}^{(0)} u \mathcal{K}+\sum_{k+|\mathcal{K}|=1}^{N-1} P_{\mathcal{K}}^{(k)} x^{k} u \mathcal{K} .
$$

Observe that the $n$-vector $P^{N}(x, u)$ is a polynomial in $x$ and $u$ without constant terms.

Let us make a transformation of the form

$$
y=z+P^{N}(x, U), \quad U \equiv U\left(x, x_{0}, u^{0}\right) .
$$

Then, the differential equation satisfied by $z$ is written as

$$
x z^{\prime}=g(x, U, z),
$$

where

$$
\begin{aligned}
& g(x, u, z)=f\left(x, z+P^{N}(x, u)\right) \\
& -x \frac{\partial}{\partial x} P^{N}(x, u)-\frac{\partial}{\partial u} P^{N}(x, u) \cdot x u^{\prime} .
\end{aligned}
$$

Here, the expression $x u u^{\prime}$ must be replaced by the expression appearing in the right-hand member of the equation ( $R$ ).

Hence, we can assume, without loss of generality, that the $n$-vector $g(x, u, z)$ is a function holomorphic and bounded in $(x, u, z)$ for

$$
|x|<a_{0}, \quad\|, u\|<b_{0}, \quad\|z\|<b_{1}
$$

for suitably chosen positive constants $a_{0}, b_{0}$ and $b_{1}$.

The equation (9.3) admits a formal solution of the form

$$
z \sim \mathbf{1}_{n}(U) \sum_{|\mathcal{K}| \geqq N-1} P_{\mathcal{K}}^{(0)} U \mathcal{K}+\sum_{k+|\mathcal{K}| \geqq N, k \geqq 1} P_{\mathcal{K}}^{(k)} x^{k} U \mathcal{K}
$$

From this fact we can verify that the n-vector $g(x, u, z)$ satisfies the vectorial inequalities

$$
\begin{aligned}
& \langle g(x, u, z)\rangle \leqq A\left(|x| \mathbf{1}_{n}+E(u)\right)\langle z\rangle \\
& \quad+B_{N}\left\{\left(|x|^{N}+|x|\|u\|^{N-1}\right) \mathbf{1}+|u \mathscr{M}|\|u\|^{N-1}\langle u\rangle\right\}, \\
& \langle g(x, u, z)-g(x, u, \tilde{z})\rangle \leqq A\left(|x| \mathbf{1}_{n}+E(u)\right)\langle z-\hat{z}\rangle
\end{aligned}
$$

for $(x, u, z)$ and $(x, u, \widetilde{z})$ in the domain (9.5), provided that the $n$ vectors $z$ and $\tilde{z}$ satisfy the vectorial inequalities 


$$
\langle z\rangle,\langle\tilde{z}\rangle \leqq K(|x| \mathbb{1}+\langle u\rangle), \quad(K>0) .
$$

Here, $A$ is a suitably chosen positive constant independent of $(x, u, z, N)$, while $B_{N}$ may depend on $N . E(u)$ is an n-by-n matrix satisfying the vectorial inequality

$$
E(u) \leqq|u \mathscr{M}| \mathbb{1}_{n}(\langle u\rangle) E \mathbb{1}_{n}(\langle u\rangle)^{-1},\|E(u)\|=\mathbb{O}(\|u\||\mathscr{M}|),
$$

where $E$ is an n-by-n constant matrix whose components are nonnegative numbers.

$\mathbb{P}$ rod Since the $n$-vector $g(x, u, z)$ is given by $(9.4)$, the terms depending on $z$ appearing in the vector $\langle g(x, u, z)\rangle$ does not exceed, in vectorial inequality,

$$
A_{2}\left(\langle F(y)\rangle+|x| \mathbb{1}_{n}\right)\langle z\rangle \text { with } y=P^{N}(0, u),\left(A_{2}>0\right)
$$

under the condition (9.8), if $|x|$ and $\|u\|$ are small. Here, the $n$-by$n$ matrix $F(y)$ is defined by (1.4). However, since $\left\langle P^{N}(0, u)\right\rangle \leqq A_{3}\langle u\rangle$ $\left(A_{3}>0\right)$, we see, by virtue of the identity (8.4) with $\langle p\rangle=A_{3}\langle u\rangle$, that there exists an $n$-by-in matrix $E(u)$ satisfying the vectorial inequalities (9.6), (9.7) and (9.9).

The last expression appearing in the vectorial inequality (9.6) is an immediate consequence of the vectorial inequality (8.3). Q.E.D.

The condition (9.8) will be naturally satisfied because we are asking the existence of a solution admitting the asymptotic expansion $\left(\mathrm{F}_{N}\right)$.

9.2. In order to prove Theorem A.3, it is sufficient to prove the following theorem:

Theorem 9.1. (Auxiliary theorem). The equation (9.3) admits one and only one solution $z=\Phi^{N}(x, U)$, where the n-vector $\Phi^{N}(x, u)$ is a function holomorphic and bounded in $(x, u)$ for

(9. 10) $)_{N} \quad|x|<a_{N}^{\prime \prime}, \quad\|\imath\|<b_{N}^{\prime \prime}, \quad \imath \mathfrak{N} \in \mathbb{D}\left(c_{N}^{\prime \prime}\right)$

and satisfying there the vectorial inequality

$$
\left\langle\Phi^{N}(x, u)\right\rangle \leqq K_{N}\left\{\left(|x|^{N}+|x|\|\cdot\|^{N-1}\right) \mathbb{1}+\|u\|^{N-1}\langle u\rangle\right\} .
$$

Here, $K_{N}$ is a certain positive constant such that 


$$
K_{N}\left\{\left(a_{N}^{\prime \prime}\right)^{N}+\left(a_{N}^{\prime \prime}\right)\left(b_{N}^{\prime \prime}\right)^{N-1}+\left(b_{N}^{\prime \prime}\right)^{N}\right\} \leqq b_{1} .
$$

In fact, by using this theorem Theorem, A. 3 can be proved in a following way: $S^{N}(x, U)=\Phi^{N}(x, U)+P^{N}(x, U)$ is a solution of the equation (1.1). Then, we can assert that, for any $N^{\prime}>N$, the identity $S^{N \prime}(x, u) \equiv S^{N}(x, u)$ holds if $(x, u)$ belongs to the common purt of the domains $(9.10)_{N^{\prime}}$ and $(9.10)_{N}$. Indeed, since the expression $z=S^{N^{\prime}}(x, U)-P^{N}(x, U)$ is obviously a solution of the equation (9.3) satisfying the condition $\langle z\rangle=O\left(\left(|x|^{N}+|x|\|U\|^{N-1}\right) \dot{1}+\|U\|^{N-1}\langle U\rangle\right)$, provided that $(x, U)$ is in the common part of these two domains. However, such a solution must be equal to $\Phi^{N}(x, U)$, which proves our assertion.

Both sides of the identity $S^{N^{\prime}}(x, u) \equiv S^{N}(x, u)$ are holomorphic functions of $x$ and $u$. Therefore, by the analytical continuation, this identity must be satisfied for $(x, u)$ in the union of the domains $(9.10)_{N^{\prime}}$ and $(9.10)_{N}$.

Let $S(x, u) \equiv S^{N}(x, u)$. Then, we can assert that the $n$-vector function $S(x, u)$ is defined in the domain

$$
|x|<a^{\prime \prime}, \quad\|u\|<b^{\prime \prime}, \quad u \mathscr{M} \in \mathfrak{D}\left(c^{\prime \prime}\right)
$$

with

$$
a^{\prime \prime}=\sup a_{N}^{\prime \prime}, \quad b^{\prime \prime}=\sup b_{N}^{\prime \prime}, \quad c^{\prime \prime}=\sup c_{N}^{\prime \prime} .
$$

So, for the proof of Theorem A.3, it is sufficient to prove that the $n$-vector $S(x, u)$ admits the asymptotic expansion (g), i. e. satisfies the inequality (A.20) in Introduction.

Let $N^{\prime}>N$. Then, we have

$$
\begin{gathered}
\left\langle S(x, u)-u-\mathbf{1}_{n}(u) \sum_{|\mathcal{K}|\left\langle N^{\prime}-1\right.} P_{\mathcal{K}^{(0)}}^{(0)}-\sum_{k+|\mathcal{K}|<N^{\prime}} P_{\left.\mathcal{K}^{(k)} x^{k} u \mathcal{K}\right\rangle}\right. \\
\leqq K_{N^{\prime}}\left(\left(|x|^{N^{\prime}}+|x|\|u\|^{N^{\prime-1}}\right) \mathbf{1}+\|u\|^{N^{\prime-1}}\langle u\rangle\right) .
\end{gathered}
$$

The norm of the vector appearing in the right-hand member does not exceed the expression $K_{N^{\prime}}\left(|x|^{N^{\prime-1}}+\|\imath\|^{N^{\prime-1}}\right)$ provided that $|x|+\|\imath\|<1$. On the other hand, there exists a positive constant $C$ such that $\left\|\mathbb{1}_{n}(u) \sum_{N-1 \leqq !} \sum_{\mathcal{K} \mid \leqq N^{\prime}-2} P_{\mathcal{K}}^{(0)} u \mathcal{K}+\sum_{N \leqq k+|\mathcal{K}|<N^{\prime}} P_{\mathcal{K}}^{(k)} x^{k} u \mathcal{K}\right\| \leqq C\left(|x|^{N}+\|u\|^{N^{\prime}}\right)$, 
Therefore, we see that the expression appearing in the left-hand member of (A.20) does not exceed the expression

$$
K_{N^{\prime}}\left(|x|^{N \prime-1}+\|u\|^{N^{\prime-1}}\right)+C\left(|x|^{N}+\|u\|^{N}\right) \leqq K_{N}\left(|x|^{N}+\|u\|^{N}\right) .
$$

This proves the inequality (A.20).

Therefore, for the proof of Theorem A.3, it is sufficient to prove Theorem 9.1.

\section{Proof of Theorem 9.1 (the Auxiliary theorem)。}

Let $\mathfrak{F}=\{\varphi(x, u)\}$ be the family of the $n$-vectors $\varphi(x, u)$ which are functions holomorphic and bounded in $(x, u)$ for the domain $(9.10)_{N}$ and satisfy there the vectorial inequality

$$
\langle\varphi(x, u)\rangle \leqq K_{N}\left\{\left(|x|^{N}+|x|\|u\|^{N-1}\right) \mathbb{1}+\|u\|^{N-1}\langle u\rangle\right\} .
$$

Let $\left(x_{0}, u^{0}\right)$ be arbitrary values in the domain $(9.10)_{N}$ and define the $n$-vector $\bar{\varphi}(x, u)$ by the formula

$$
\bar{\varphi}\left(x_{0}, u^{0}\right)=\int_{0}^{x_{0}} g(x, U, \varphi(x, U)) \frac{d x}{x},
$$

where the integration should be taken along the path $\Gamma_{x_{0}}$ which has been already defined in Section 6 . Then, the mapping $\mathfrak{I}$ is defined as follows:

$$
\varphi(x, u) \stackrel{\mathfrak{I}}{\longrightarrow} \bar{\varphi}(x, u) .
$$

The family $\mathfrak{F}$ is not empty since $\{0\} \in \mathfrak{F}$ and $\mathfrak{F}$ is convex, closed and normal. On the other hand, by virtue of Theorem 6.2 , as $x$ is on the path $\Gamma_{x_{0}}$ the values of $x$ and $U\left(x, x_{0}, u^{0}\right)$ remain in the domain $(9.10)_{N}$ if the initial values $x_{0}$ and $u^{0}$ belong to the same domain. Moreover, as we shall show later, the integral (10.2) converges uniformly with respect to $u^{0}$ for each $x_{0}$. This assures us that the mapping $\mathfrak{I}$ has a well-defined meaning.

Our proof of the auxiliary theorem is based on the existence of a fixed point of this mapping (See, for example, M. Hukuhara [3]).

We shall first prove the following two propositions:

Proposition 10.1. The mapping $\mathfrak{I}$ transforms $\mathfrak{F}$ into itself. 
This proposition is equivalent to the following three assertions:

(a) The integral (10.2) is convergent.

(b) We have the vectorial inequality

$$
\left\langle\bar{\varphi}\left(x_{0}, u^{0}\right)\right\rangle \leqq K_{N}\left\{\left(\left|x_{0}\right|^{N}+\left|x_{0}\right|\left\|u^{0}\right\|^{N-1}\right) 1+\left\|u^{0}\right\|^{N-1}\left\langle u^{0}\right\rangle\right\} .
$$

(c) The n-vector $\bar{\varphi}(x, u)$ is a function holomorphic and bounded in $(x, u)$ for the domain $(9.10)_{N}$.

Proposition 10.2. I is a continuous mapping of $\mathfrak{F}$ with respect to the topology of uniform convergence.

This results immediately from the following assertion:

(d) If the sequence $\left\{\varphi^{m}(x, u)\right\}$ tends to 0 with respect to the topology of $\mathfrak{F}$, then the corresponding sequence $\left\{\bar{\varphi}^{n}(x, u)\right\}$ also tends to 0 .

Suppose that these two propositions have been established. Then, by means of a fixed point theorem, it is concluded that there exists a fixed point of the mapping $\mathfrak{I}$, namely, a member $\varphi(x, u) \in \mathfrak{F}$ such that $\varphi(x, u)=\bar{\varphi}(x, u)$. We denote it by $\Phi^{N}(x, u)$.

Then, we can prove the following:

Proposition 10.3. The n-vector $\Phi^{N}(x, U)$ is a solution of the equation (9.3).

For the proof of this proposition, it is sufficient to show that:

(e) We have the identity

$$
\frac{d}{d x_{1}} \bar{\varphi}\left(x_{1}, u^{1}\right)=G\left(x_{1}, u^{1}\right)
$$

where $u^{1}$ is a function $x_{1}$ defined be $u^{1}=U\left(x_{1}, x_{0}, u^{0}\right)$, and

$$
G(x, u)=g(x, u, \varphi(x, u)) \frac{1}{x} .
$$

Our final proposition is stated as follows:

Proposition 10.4. The solution of the equation (9.3) satisfying the condition

$$
z=O\left(\left(|x|^{N}+|x|\|U\|^{N-1}\right) \mathbf{1}+\|U\|^{N-1}\langle U\rangle\right)
$$

is unique. 
This proposition results from the following assertion:

(i) The quantity $K$ defined by

$$
K=\max _{j=1}^{n} \sup _{x, u}\left\{\left|\phi_{j}(x, u)\right|\left(|x|^{N}+|x|\|u\|^{N-1}+\|u\|^{N-1}\left|u_{j}\right|\right)^{-1}\right\}
$$

is equal to 0 , where $\phi(x, U)$ is the difference of two solutions satisfying the same conditions.

\section{Proof of the assertions $(\mathbf{a}) \sim(\mathbf{f})$.}

11.1. The assertion (a). If we substitute the expression appearing in the right-hand member of $(10.1)_{N}$ for $z$, then the vectorial inequality (9.6) is reduced to

$$
\langle g(x, u, \varphi(x, u))\rangle \leqq \mathcal{G}(x, u),
$$

where the $n$-vector $\mathcal{G}(x, u)$ is given by

$$
\begin{aligned}
& \mathcal{G}(x, u)=|x|^{N}\left\{\left(A K_{N}|x|+B_{N}\right) \mathbb{1}+A K_{N} E(u) \mathbb{1}\right\} \\
& \quad+|x|\|u\|^{N-1}\left\{\left(A K_{N}|x|+B_{N}\right) \mathbb{1}+A K_{N}\langle u\rangle+A K_{N} E(u) \mathbb{1}\right\} \\
& \quad+\|u\|^{N-1}\left\{B_{N}|u \mathscr{M}| \mathbb{1}_{n}+A K_{N} E(u)\right\}\langle u\rangle .
\end{aligned}
$$

We change the integration variable from $x$ to $w=U \mathscr{M}$ by the first relation of $\left(\mathrm{R}^{\prime}\right)$ in Section 5. Then, we have the vectorial inequality

$$
\left\langle\bar{\varphi}\left(x_{0}, u^{0}\right)\right\rangle \leqq\left|\int_{\Gamma_{w 0}^{*}} \mathcal{G}(\tilde{x}(w), \widetilde{U}(w)) \frac{d w}{w^{2} X(w, \widetilde{U}(w))}\right|,
$$

where

$$
X(w, \widetilde{U}) \equiv(\mathscr{M} \cdot \boldsymbol{\alpha})+\left(\mathscr{M} \cdot \boldsymbol{\alpha}^{*}\right) w=O(|\mathscr{M} \cdot \boldsymbol{\alpha}|) .
$$

Here, $\tilde{x}(w)$ and $\tilde{U}(w)$ are the same as those appearing in Section 5 .

As we have already seen in Sections 6 and 7,w tends to 0 along the path $\Gamma_{w 0}^{*}$ as $x$-tends to 0 along the path $\Gamma_{x_{0}}$, and the part of the path $\Gamma_{w 0}^{*}$, which is sufficiently near $w=0$, consists of the segment that was denoted by $\Gamma^{\prime \prime}$.

As can be easily seen from the formula (5.4), we have

$$
\|\tilde{U}(w)\|=O\left(|w|\left\|\operatorname{Re} \frac{\alpha}{(\mathscr{M} \cdot \alpha)}\right\|^{\prime}\right) \quad \text { for } w \in \Gamma^{\prime \prime}
$$


and the function $\tilde{x}(w)$ tends exponentially to 0 as $w$ approaches 0 along the segment $\Gamma^{\prime \prime}$.

Since, by (9.9), we have

$$
\|E(\widetilde{U}(w))\| \leqq C_{1}\|\widetilde{U}(w)\|^{|\mathscr{M}|}, \quad C_{1}>0
$$

the norm of the vector $\mathcal{G}(\tilde{x}(w), \widetilde{U}(w))$ does not exceed the expression $C_{2}\left(|\tilde{x}(w)|^{N}+x|(w)|\|\tilde{U}(w)\|^{N-1}+\|\tilde{U}(w)\|^{N}\right)\left(C_{2}>0\right)$. Therefore, by virtue of the condition (11.5), we see that, if $N$ is large enough to satisfy the inequality

$$
N\left\|\operatorname{Re} \frac{\boldsymbol{\alpha}}{(\mathscr{M} \cdot \boldsymbol{\alpha})}\right\|>1
$$

the integral in (11.3) and, consequently, the integral (10.2) is convergent.

11.2. The assertion (b). We can assume, without loss of generality, that

$$
|X(w, \widetilde{U}(w))| \geqq \frac{1}{2}|(\mathscr{M} \cdot \boldsymbol{\alpha})|
$$

for $\widetilde{U}(w)$ in the domain $(9.10)_{N}$.

Let $s$ and $s_{0}$ be the length of the curve $\Gamma_{w 0}^{*}$ measured from the origin to the points $w$ and $w^{0}$ respectively. Then, we have $\left|\frac{d w}{d s}\right|=1$ for $w_{\in} \in \Gamma_{w^{0}}^{*}$. Therefore, by (11.3), we have the vectorial inequality

$$
\left\langle\bar{\varphi}\left(x_{0}, u^{0}\right)\right\rangle \leqq \frac{2}{|(\mathscr{M} \cdot \alpha)|} \int_{0}^{s_{0}} \mathcal{G}(\tilde{x}(w), \widetilde{U}(w)) \frac{d s}{|w|^{2}} .
$$

To obtain the inequality (10.4), it is sufficient to prove that the vectorial inequality

$$
\begin{aligned}
& \frac{2}{|(\mathscr{M} \cdot \boldsymbol{\alpha})|} \int_{0}^{s} \mathcal{G}(\tilde{x}(w), \tilde{U}(w)) \frac{d s}{|w|^{2}} \\
& \leqq K_{N}\left\{\left(|\tilde{x}(w)|^{N}+|\tilde{x}(w)|\|\tilde{U}(w)\|^{N-1}\right) \mathbb{1}+\|\tilde{U}(w)\|^{N-1}\left\langle\tilde{U}\left(w^{\prime}\right)\right\rangle\right\}
\end{aligned}
$$

is satisfied for every $s\left(0 \leqq s \leqq s_{0}\right)$ or, what is the same thing, that we have the vectorial inequality

$$
\begin{aligned}
& (11.10)^{\prime} \frac{2}{|(\mathscr{M} \cdot \boldsymbol{\alpha})|} \mathcal{G}(\tilde{x}(w), \widetilde{U}(w)) \frac{1}{|w|^{2}} \\
& \leqq K_{N} \frac{d}{d s}\left\{\left(\left|\tilde{x}\left(w^{\prime}\right)\right|^{N}+|\tilde{x}(w)|\|\tilde{U}(w)\|^{N-1}\right) \mathbf{1}+\|\tilde{U}(\mathfrak{w})\|^{N-1}\langle\widetilde{U}(w)\rangle\right\} .
\end{aligned}
$$


Now we shall show that the vectorial inequality $(11.10)^{\prime}$ is satisfied for the domain $(9.10)_{N}$.

Owing to the Theorem 6.1 and Theorem 7.1 , we have the inequalities

$$
\left\{\begin{aligned}
& \frac{d}{d s}\left(|\tilde{x}(w)|^{N}\right) \geqq \frac{N \sin \delta_{0}}{2|(\mathscr{M} \cdot \alpha)||w|^{2}}|\tilde{x}(w)|^{N}, \\
& \frac{d}{d s}\left(|\tilde{x}(w)|\|\tilde{U}(w)\|^{N-1}\right) \geqq \frac{\left(1+(N-1)|w|\|\alpha\|^{\prime}\right) \sin \delta_{0}}{2|(\mathscr{M} \cdot \alpha)||w|^{2}}|\tilde{x}(w)|\|\widetilde{U}(w)\|^{N-1}, \\
& \frac{d}{d s}\left(\|\widetilde{U}(w)\|^{N-1}\langle\widetilde{U}(w)\rangle\right) \geqq \frac{N\|\alpha\|^{\prime} \sin \delta_{0}}{2|(\mathscr{M} \cdot \alpha) !| w \mid}\|\widetilde{U}(w)\|^{N-1}\langle\widetilde{U}(w)\rangle .
\end{aligned}\right.
$$

Therefore, for the proof of the vectorial inequality $(11.10)^{\prime}$, we have only to get the vectorial inequality:

$$
\begin{aligned}
& 4 \mathcal{G}(\tilde{x}, \tilde{U}) \leqq K_{N}\left\{\left(N \sin \delta_{0} \cdot|\tilde{x}|^{N}+\sin \delta_{0} \cdot|\tilde{x}|\|\tilde{U}\|^{N-1}\right) \mathbb{1}\right. \\
& \left.+N\|\alpha\|^{\prime} \sin \delta_{0} \cdot|\tilde{U} \mathscr{M}|\|\tilde{U}\|^{N-1}\langle\tilde{U}\rangle\right\},
\end{aligned}
$$

if we notice that $w \equiv \tilde{U}(w) \mathscr{M}$. However, since the $n$-vector $\mathcal{L}(x, u)$ is defined by (11.2) and we have the inequality (11.6), the vectorial inequality (11.12) results from the following three inequalities:

$$
\begin{aligned}
& 4\left\{A K_{N}\left(|x|+C_{1}\|u\||\mathcal{S}|+B_{N}\right\} \leqq K_{N} N \sin \delta_{0},\right. \\
& 4\left\{A K_{N}\left(|x|+C_{1}\|u\||\mathcal{M}|+\|u\|\right)+B_{N}\right\} \leqq K_{N} \sin \delta_{0}, \\
& 4\left(A K_{N} C_{2}+B_{N}\right) \leqq K_{N} N\|\alpha\|^{\prime} \sin \delta_{0} \quad(\text { by } w \equiv \widetilde{U}(w) \mathscr{M}) .
\end{aligned}
$$

The last inequality is obtained by the fact that there exists a positive constant $C_{2}$ such that

$$
E(\tilde{U})\langle\widetilde{U}\rangle \leqq C_{2} \mid \widetilde{U} \mathscr{A l}\langle\tilde{U}\rangle,
$$

because of the vectorial inequalities (8.6) and (9.9). The inequality (11.13) is automatically satisfied if the inequality (11.14) is satisfied.

We take first the quantities $1 / N, a_{N}^{\prime \prime}$ and $b_{N}^{\prime \prime}$ so small that

$$
\left\{\begin{array}{l}
8 A\left(a_{N}^{\prime \prime}+C_{1}\left(b_{N}^{\prime \prime}\right)|\mathcal{I}|+b_{N}^{\prime \prime}\right) \leqq \sin \delta_{0}, \\
8 A C_{2} \leqq N\|\alpha\|^{\prime} \sin \delta_{0}
\end{array}\right.
$$


and next $K_{N}$ sufficiently large so that the inequalities (11.13), (11.14) and (11.15) are satisfied for the values of $\tilde{x}(w)$ and $\tilde{U}(w)$ in the domain $(9.10)_{N}$. This is evidently possible. Moreover, for the positive constant $K_{N}$ thus determined, by taking, if necessary, the values of $a_{N}^{\prime \prime}$ and $b_{N}^{\prime \prime}$ much smaller, the inequality (9.12) also is satisfied.

11.3. The assertion (c). The inequality (10.4) shows us that the integral (10.2) is uniformly convergent with respect to $u^{0}$ for each $x_{0}$. Therefore, if we could prove that, for any $x_{1}$ sufficiently near to $x_{0}$, the relation

$$
\int_{\Gamma_{x_{0}}} g(x, U, \varphi(x, U)) \frac{d x}{x}=\int_{\Gamma_{x_{1}}} g \frac{d x}{x}+\int_{x_{1}}^{x_{0}} g \frac{d x}{x}
$$

holds, the assertion (c) would follow from the Lemma 1 in Section 25 in M. Iwano [1].

Here, the last integration should be taken along the segment joining $x_{0}$ with $x_{1}$, and the curve $\Gamma_{x_{1}}$, is an image of the curve $\Gamma_{w 1}^{*}$ by the transformation defined by the first relation of (5.4), where $\hat{c}$ must be so chosen that $\tilde{x}\left(w^{0}\right)=x_{0}$. Of course,

$$
w^{1}=U\left(x_{1}, x_{0}, u^{0}\right) \pi
$$

We notice that the point $w$ on the curve $\Gamma_{w i}^{*}(i=0,1)$ is on the segment denoted by $\Gamma_{i}^{\prime \prime}(i=0,1)$ and is located in the sector

$$
\theta_{-}+2 \delta_{0} \leqq \arg w \leqq \theta_{+}-2 \delta_{0}
$$

if $|w|$ is sufficiently small.

Let $\xi_{i}(\rho)(i=0,1)$ be the points of intersection of a sufficiently small circle $|w|=\rho$ with the paths $\Gamma_{w i}^{*}(i=0,1)$. Then, the function $\max |\tilde{x}(w)|$ for $w \in \overparen{\xi_{0}(\rho) \xi_{1}}(\rho)$ tends exponentially to zero with $\rho$ and we have

$$
\max \|\widetilde{U}(w)\|=O\left(\rho^{\left\|\operatorname{Re} \frac{\alpha}{(\mathscr{N} \cdot \alpha)}\right\|} \|^{\prime}\right) \rightarrow 0 \quad(\text { as } \rho \rightarrow 0) .
$$

Therefore, as can be easily seen from the proof of the assertion (a), it is concluded that: 


$$
\left\|\int_{\xi_{0}(\rho)}^{\xi_{1}(\rho)} \frac{g(\tilde{x}(w), \tilde{U}(w), \varphi(\tilde{x}(w), \tilde{U}(w))}{w^{2} X(w, \tilde{U}(w))} d w\right\| \rightarrow 0 \quad \text { as } \rho \rightarrow 0 .
$$

Here, the integration must be taken along the circle $|w|=\rho$.

Let $x_{0} \overparen{(\rho) x_{1}}(\rho)$ be the image of the curve $\overbrace{\xi_{0}}(\rho) \xi_{1}(\rho)$ such that $x_{i}(\rho) \in \Gamma_{x_{i}}(i=0,1)$ by the same transformation as before. Then, the relation (11.18) is equivalent to the fact that

$$
\left\|\int_{x_{0}(\rho) x_{1}(\rho)} g(x, U, \varphi(x, U)) \frac{d x}{x}\right\| \rightarrow 0 \quad \text { as } \rho \rightarrow 0 .
$$

As $\rho$ tends to 0 , the points $x_{i}(\rho)(i=0,1)$ tend to the origin. Hence, the relation (11.19) proves the identity (11.17).

11. 4. The assertion (d). Since the topology of $\widetilde{F}$ is defined by uniform convergence, as can be easily seen from the proof of the vectorial inequality (10.4), this assertion is almost obvious.

11.5. The assertion (e). Since, by definition, we have

$$
\bar{\varphi}\left(x_{1}, u^{1}\right)=\int_{0}^{x_{1}} G\left(x, U\left(x, x_{1}, u^{1}\right)\right) d x, \quad \text { (See (10.6)). }
$$

By virtue of the assertion ( $\mathfrak{c}$ ), we can apply the formula of differentiation under the sign $\int$. Therefore, we find that:

$$
\begin{aligned}
& \frac{d}{d x_{1}} \bar{\varphi}\left(x_{1}, u^{1}\right)=G\left(x_{1}, u^{1}\right) \\
& +\int_{0}^{x_{1}} \frac{\partial G(x, U)}{\partial U}\left\{\frac{\partial U\left(x, x_{1}, u^{1}\right)}{\partial x_{1}}+\frac{\partial U\left(x, x_{1}, u^{1}\right)}{\partial u^{1}} \frac{\partial U\left(x_{1}, x_{0}, u^{0}\right)}{\partial x_{1}}\right\} d x .
\end{aligned}
$$

However, as is well known, $U\left(x_{0}, x_{1}, u^{1}\right)$ is an integral of the equation (R) for an arbitrary constant $x_{0}$. Hence, the expression in the braces \{ $\}$ vanishes identically, which proves the relation (10.5).

11.6. The assertion $(\mathbb{1})$. Let $\phi(x, U)$ be the difference of two solutions satisfying the same conditions. Then, by virtue of the vectorial inequality (9.7), we have

$$
\left\langle\phi\left(x_{0}, u^{0}\right)\right\rangle \leqq \frac{2 A}{|(\mathscr{M} \mid \alpha)|} \int_{0}^{s_{0}}\left(|\tilde{x}| \mathbb{1}_{n}+E(\tilde{U})\right)\langle\phi(\tilde{x}, \tilde{U})\rangle \frac{d s}{|w|^{2}}
$$


Suppose $K>0$. The definition of $K$ implies that

$$
\langle\phi(\tilde{x}, \tilde{U})\rangle \leqq K\left\{\left(|\tilde{x}|^{N}+|\tilde{x}|\|\tilde{U}\|^{N-1}\right) \mathbf{1}+\|\tilde{U}\|^{N-1}\langle\tilde{U}\rangle\right\} .
$$

Substituting the expression appearing in the right-hand member of (11.21) for $\phi(\tilde{x}, \tilde{U})$ in the vectorial inequality (11.20), as we have already proved, the integrand of the resulting expression does not exceed, in vectorial inequality, the expression

$$
\begin{gathered}
\frac{4 A K}{|(\mathscr{M} \cdot \boldsymbol{\alpha})||w|^{2}}\left\{\left(a_{N}^{\prime \prime}+C_{1}\left(b_{N}^{\prime \prime}\right)|\mathscr{M}|+b_{N}^{\prime \prime}\right)\left(|\tilde{x}|^{N}+|\tilde{x}|\|\widetilde{U}\|^{N-1}\right) \mathbf{1}\right. \\
\left.+C_{2}|\widetilde{U} \mathscr{M}|\|\widetilde{U}\|^{N-1}\langle\widetilde{U}\rangle\right\} .
\end{gathered}
$$

Integrating the vectorial expression above with respect to $s$ from 0 to $s_{0}$, we have the vectorial inequality

$$
\begin{aligned}
\left\langle\phi\left(x_{0}, u^{0}\right)\right\rangle & \leqq \frac{4 A K\left(a_{N}^{\prime \prime}+C_{1}\left(b_{N}^{\prime \prime}\right)|\mathscr{M}|+b_{N}^{\prime \prime}\right)}{N \sin \delta_{0}}\left(\left|x_{0}\right|^{N}+\left|x_{0}\right|\left\|u^{0}\right\|^{N-1}\right) \mathbb{1} \\
& +\frac{4 A K C_{2}}{N\|\alpha\| \|^{\prime} \sin \delta_{0}}\left\|u^{0}\right\|^{N-1}\left\langle u^{0}\right\rangle
\end{aligned}
$$

because of the inequalities (11.11). However, by the inequalities (11.16), the above inequality implies that:

$$
\left\langle\phi\left(x_{0}, u^{0}\right)\right\rangle \leqq \frac{K}{2}\left\{\left(\left|x_{0}\right|^{N}+\left|x_{0}\right|\left\|u^{0}\right\|^{N-1}\right) \mathbb{1}+\left\|u^{0}\right\|^{N-1}\left\langle u^{0}\right\rangle\right\} .
$$

From this it follows that $2 K \leqq K$, which contradicts our hypothesis. Hence, we must have $K=0$.

\section{Chapter IV. Proof of Theorem A.2.}

\section{The differential equations which determine the $n$-vectors} $P^{(k)}(u)(k \geqq 0)$.

Let $U$ be the same as that in Section 8. As we have already proved, the formal power series

$$
y^{\prime} \sim U+\mathbb{1}_{n}(U) P^{(0)}(U)+\sum_{k=1}^{\infty} P^{(k)}(U) x^{k},
$$

which is obtained by replacing $u$ by $U$ in the power series (f) appearing in Theorem A.1, is a formal solution of the equation (1.1). 
By differentiating both sides of the formal solution $\left(\mathrm{F}^{*}\right)$ term by term, we obtain formally

$$
\begin{aligned}
& y \mathcal{M} \mathbf{1}_{n}(y) f^{(0)}(y)+\sum_{k=1}^{\infty} f^{(k)}(y) x^{k} \sim x U^{\prime} \\
& \quad+\mathbf{1}_{n}\left(x U^{\prime}\right) P^{(0)}(U)+\mathbf{1}_{n}(U) x\left(P^{(0)}(U)\right)^{\prime} \\
& \quad+\sum_{k=1}^{\infty}\left\{k P^{(k)}(U)+x\left(P^{(k)}(U)\right)^{\prime}\right\} x^{k}, \quad\left({ }^{\prime}=\frac{d}{d x}\right)
\end{aligned}
$$

with identities

$$
x\left(P^{(k)}(U)\right)^{\prime} \equiv \frac{\partial P^{(k)}(U)}{\partial U} x U^{\prime} \quad(k \geqq 0),
$$

where $x U^{\prime}$ must be replaced by the expression in the right-hand member of the simplified equation $(\mathrm{R})$ with $u=U$. Hence, $x\left(P^{(k)}(U)\right)^{\prime}$ can be regarded as functions of $U$ alone.

Substituting the formal solution $\left(\mathrm{F}^{*}\right)$ for $y$ appearing in the lefthand side of (12.1), the equation (12.1) can be reduced to a formal identity between formal power series of $x$ whose coefficients are also formal power series of $U$. Hence, comparing the coefficients of the terms $x^{k}(k=0,1, \cdots)$ of this formal identity, we can find differential equations satisfied by the $n$-vector functions $P^{(k)}(U)(k=0,1, \cdots)$.

By a simple calculation, we see that these differential equations are given by the formula (A.14) or $(\text { A.17) })^{k}$ in Introduction according as we have $k=0$ or $k \geqq 1$. More precisely, the $n$-vector $R^{(k)}(u)$ appearing in the equation (A.17) $)^{k}$ is expressed by the sum of the $n$-vector $f^{(k)}\left(u+\mathbb{1}_{n}(u) P^{(0)}(u)\right)$ and a linear form of the $n$-vectors

$$
\begin{aligned}
& \frac{\partial \mathcal{K}}{\partial z^{\mathcal{K}}} \hat{f}^{(j)}\left(u+\mathbb{1}_{n}(u) P^{(0)}(u)\right) \cdot P^{(i)}(u) \mathcal{K} \\
& \left(i|\mathcal{K}|+j \leqq k, \quad 1 \leqq i \leqq k-1, \hat{f}^{(\jmath)}(z) \equiv z^{\mathcal{M}} \mathbb{1}_{n}(z) f^{(0)}(z)(j=0) \text { or } f^{(j)}(z)\right. \\
& (j \geqq 1)) .
\end{aligned}
$$

Moreover, these differential equations possess formal solutions of the form

$$
P^{(k)} \sim \sum_{\mathcal{K}} P_{\mathcal{K}}^{(k)} U^{\mathcal{K}}
$$

which is obtained by replacing $u$ by $U$ in the power series $(\mathrm{A} .10)^{k}$ in Introduction. 
13. Determination of the $n$-vector function $P^{(0)}(u)$.

13. 1. We consider the differential equation (A.14) and its formal solution (12.2) ${ }^{0}$. Observe that the expression appearing in the righthand member of (12.2) is developable in a uniformly convergent power series of $P$ whose coefficients are functions developable in uniformly convergent power series of the $n$-vector $U$. Hence, the equation (A.14) can be written as

$$
x P^{\prime}=U \mathscr{M h}(U, P),
$$

where $h(u, p)$ is an $n$-dimensional column vector function holomorphic and bounded in $(u, p)$ for

$$
\|u\|<b_{0},\|p\|<d_{0} .
$$

As we have already seen, this equation admits the formal solution $(12.2)^{0}$ :

$$
P \sim \sum_{|\mathcal{K}| \geqq 1} P_{\mathcal{K}}^{(0)} U \mathcal{K}
$$

We shall prove that the power series (13.2) is uniformly convergent for $\|U\|<b_{0}^{\prime \prime}$ and represents a solution of the equation (13.1).

Instead of proving the uniform convergence of the formal solution (13.2) directly, we will prove the uniform convergence of the power series

$$
P \sim \sum_{|\mathcal{K}| \geqq 1} P_{\mathcal{K}}^{(0)} V^{\mathcal{K}}
$$

Here, $V \equiv V\left(x, x_{0}, v^{0}\right)$ is the holomorphic solution of the differential equation

$$
x(\mathscr{H} \cdot X(v)) v^{\prime}=\mathbf{1}_{n}(X(v)) v
$$

with

$$
X(v)=\alpha+\sum_{\mathscr{H} \in \mathscr{S}} \beta_{\mathscr{H}} v^{\mathscr{H}}
$$

satisfying the initial condition $V=v^{0}$ at $x=x_{0}$, where $x_{0}$ and $v^{0}$ are arbitrary values such that

$$
\left|x_{0}\right|<a_{0}^{\prime \prime}, \quad\left\|v^{0}\right\|<b_{0}^{\prime \prime} .
$$


Then, we can prove the following proposition:

Proposition 13.1. The formal power series (13.2*) is a formal solution of the differential equation

$$
x(\mathscr{M} \cdot X(V)) P^{\prime}=h(V, P) .
$$

Conversely, let $P=g(V)$ be the actual solution of the equation (13.1*). Then, the n-vector $g(U)$ is a solution of the equation (13.1).

Proof. Let $g(U)$ be the $n$-vector expressed by the formal power series (13.2). Then, since $g(U)$ satisfies formally the equation (13.1), we have the formal identity

$$
\frac{\partial g(U)}{\partial U} x U^{\prime}=U \Re h(U, g(U))
$$

with

$$
\frac{\partial g(U)}{\partial U} \sim \sum_{\mathcal{K}} U \mathcal{K} P_{\mathcal{K}}^{(0)} \mathcal{K} \mathbb{1}_{n}(U)^{-1},
$$

which, by virtue of the equation (R), implies that:

$$
U \mathscr{M} \frac{\partial g(U)}{\partial U} \mathbb{1}_{n}(X(U)) U=U \mathscr{M h}(U, g(U)) \text {. }
$$

From this, we see that the $n$-vector $g(u)$ satisfies formally the partial differential equation

$$
\frac{\partial g}{\partial u} \mathbb{1}_{n}(X(u)) u=h(u, g)
$$

Owing to this equation, it is readily seen, by a direct calculation, that the $n$-vector $g(V)$ is a formal solution of the equation $\left(13.1^{*}\right)$.

Suppose that the $n$-vector $g(V)$ is the actual solution of the equation $\left(13.1^{*}\right)$. Then, a short calculation shows us that the $n$-vector $g(v)$ must satisfy the partial differential equation (13.4) with $u=v$. This fact means that the $n$-vector $g(U)$ is an actual solution of the equation (13. 1).

Q.E. D.

13.2. In order to prove the uniform convergence of the formal solution $\left(13.2^{*}\right)$, we apply a transformation of the form

$$
P=q+P^{N}(V)
$$


to the equation $\left(13.1^{*}\right)$, where

$$
P^{\mathrm{v}}(v) \equiv \sum_{|\mathcal{K}|<N} P_{\mathcal{K}}^{(0)} v^{\mathcal{K}}
$$

Noticing that the quantity $(\mathcal{H} \cdot \alpha)$ appearing in the expression $(\mathcal{S} \cdot \mathrm{X}(v)$ ) is non-zero, the transformed diffe:ential equation can be written as

$$
x q^{\prime}=\hat{h}(V, q) .
$$

Here, $\hat{h}(v, q)$ is an $n$-dimensional column vector function ho'cmorphic and bounded in $(v, q)$ for

$$
\|v\|<b_{1}, \quad \| q^{\|} \mid<d_{1}
$$

and satisfying there the inequalities

$$
\begin{aligned}
& \|\hat{h}(v, q)\| \leqq A\|q\|+B_{N}\|v\|^{*}, \\
& \|\hat{h}(\tau, q)-\hat{h}(v, \hat{q})\| \leqq-1\|q-\hat{q}\|,
\end{aligned}
$$

if $(\vartheta, \hat{q})$ belongs to the domain (13.8). A is a positive constant independent of $(\tau, q, N)$, while $B_{N}$ may depend on $N$. Moreover, the equation (13.7) admits a formal solution of the form

$$
q \sim \sum_{|\mathcal{K}| \geqq N} P_{\mathcal{K}}^{(0)} V \mathcal{K}
$$

13.3. Let $L_{x_{0}}$ be the segment joining the point $x_{0}$ with the origin and $s$ be the length of this segment measured from the origin to the point $x$.

Then, we have the following proposition:

Proposition 13.2. We have the inequality

$$
\frac{d\|V\|}{d s} \geqq \frac{\|\alpha\|^{\prime} \sin \delta_{0}}{2 \mid(\mathscr{M} \cdot \alpha)}\|V\|
$$

on the path $L_{x_{0}}$.

Proof. We have the relation

$$
\frac{1}{x} \frac{d x}{d s}=\frac{1}{|x|} \quad \text { for } \quad x \in L_{x_{0}} .
$$

Therefore, 


$$
\begin{aligned}
& \frac{1}{\|V\|} \frac{d\|V\|}{d s} \geqq \min _{j} \operatorname{Re}\left(\frac{x}{V_{j}} \frac{d V_{j}}{d x} \frac{1}{x} \frac{d x}{d s}\right) \\
& =\frac{1}{|x|} \min _{j} \operatorname{Re}\left\{\frac{\boldsymbol{\alpha}_{j}}{(\mathscr{M} \cdot \boldsymbol{\alpha})}\left[\left(1+\frac{\sum \beta \mathcal{H}_{\mathcal{H}},{ }_{j} V_{\mathcal{H}}}{\boldsymbol{\alpha}_{j}}\right)\left(1+\frac{\sum\left(\mathscr{M} \cdot \boldsymbol{\beta}_{\mathscr{H}}\right) V \mathscr{H}}{(\mathscr{M} \cdot \boldsymbol{\alpha})}\right)^{-1}\right]\right\},
\end{aligned}
$$

if we notice that $V$ is a solution of the equation (13.3). We can choose the value of $b_{0}^{\prime \prime}$ so that the expression in the blacket is not less than $1 / 2$ in module and its argument is not greater than $\delta_{0}$ in module for $\|V\|<b_{0}^{\prime \prime}$. Hence, the inequality (6.3) yields the inequality (13.11).

Q.E. D.

We can prove the following proposition:

Proposition 13.3. The differential equation (13.7) admits one and only one solution $q=\Phi^{N}(V)$, where the n-vector $\Phi^{N}(v)$ is a function holomorphic and bounded in $v$ for $\|v\|<b_{N}^{\prime \prime}$ and satisfying there the inequality

$$
\left\|\Phi^{N}(v)\right\| \leqq K_{N}\|v\|^{N},
$$

$K_{N}$ being a certain positive constant.

Proof. Let $\mathfrak{\mho}=\{\varphi(v)\}$ be the family of the $n$-vectors $\varphi(v)$, which are functions holomorphic and bounded in $v$ for $\|v\|<b_{N}^{\prime \prime}$ and satisfying there the inequality

$$
\|\varphi(v)\| \leqq K_{N}\|v\|^{N} .
$$

Then, the mapping $\mathfrak{I}$ is defined as follows:

$$
\varphi(v) \stackrel{\mathfrak{I}}{\longrightarrow} \bar{\varphi}(v),
$$

where

$$
\bar{\varphi}\left(v^{0}\right)=\int_{L_{x_{0}}} \hat{h}(V, \varphi(V)) \frac{d x}{x} .
$$

Here, $v^{0}$ is an arbitrary value such that $\left\|v^{0}\right\|<b_{N}^{\prime \prime}$. The fact that the $n$ vector $\bar{\varphi}\left(v^{0}\right)$ does not depend on $x_{0}$ can be easily verified by changing the integration variable from $x$ to $V_{1}$ by the relation

$$
x(\mathscr{M} \cdot X(V)) V_{1}^{\prime}=\left(\alpha_{1}+\sum \beta_{\mathscr{G}, 1} V \mathscr{H}\right) V_{1},
$$


where the letters with the subindex 1 signify the first entries of the corresponding $n$-vectors.

For the proof of the Proposition 13.3, it is sufficient to prove six assertions corresponding to the assertions $(\mathbf{a}) \sim(\mathbf{f})$ in Section 10 . We consider the following correspondence:

(1) The integral (13.14) to the integral (10.2);

(2) The path $L_{x_{0}}$ to the path $\Gamma_{x_{0}}$;

(3) The inequality $(13.13)_{N}$ to the vectorial inequality $(10.1)_{N}$;

(4) Proposition 13.2 to Theorems 6.1 and 7.1;

(5) The inequalities $(13.9),(13.10)$ to the vectorial inequalities (9.6), (9.7).

Then, the proof of these six assertions is almost exactly the same as or rather simpler than that of the original assertions, because, in this case, we can replace the symbol \langle\rangle appearing in Sections $9 \sim 11$ by the norm \|\| . For example, the proof of the corresponding assertion (b):

$$
\left\|\bar{\varphi}\left(v^{0}\right)\right\| \leqq K_{N}\left\|v^{0}\right\|^{N}
$$

can be carried out as follows: By virtue of (13.9) and (13.13) $)_{N}$, we have

$$
\left\|\bar{\varphi}\left(\mathcal{v}^{0}\right)\right\| \leqq \int_{0}^{\left|x_{0}\right|}\left(A K_{N}+B_{N}\right)\|V\|^{N} \frac{d|x|}{|x|} \quad(s=|x|),
$$

which, owing to the inequality (13.11), implies that

$$
\left\|\bar{\varphi}\left(v^{0}\right)\right\| \leqq \frac{2|(\mathscr{M} \cdot \alpha)|\left(A K_{N}+B_{N}\right)}{N\|\alpha\|^{\prime} \sin \delta_{0}}\left\|v^{0}\right\|^{N} .
$$

Therefore, in order to obtain the inequality (13.15), it suffices that we have

$$
2|(\mathscr{M} \cdot \boldsymbol{\alpha})|\left(A K_{N}+B_{N}\right) \leqq N\|\boldsymbol{\alpha}\|^{\prime} \sin \delta_{0} \cdot K_{N} .
$$

We first take $N$ so large that

$$
4|(\mathscr{M} \cdot \boldsymbol{\alpha})| A \leqq N\|\boldsymbol{\alpha}\|^{\prime} \sin \boldsymbol{\delta}_{0}
$$

and next $K_{N}$ large enough to have the inequality (13.16). 
13. 4. By virtue of the Proposition 13.3, we see that

$$
P^{N}(V)+\Phi^{N}(V)
$$

is a solution of the equation $\left(13.1^{*}\right)$ and is independent of $N$. Therefore, if we denote this expression by $S(V)$, the $n$-vector $S(V)$ admits the asymptotic expansion $\left(13.2^{*}\right)$ as $V$ tends to 0 in the domain $\|v\|$ $<b_{0}^{\prime \prime}$ with $b_{0}^{\prime \prime}=\sup b_{0}^{\prime \prime}$. However, since the point $v=0$ is an inner point of the domain in which the solution $S(V)$ is defined, the function $S(v)$ is holomorphic at $v=0$. Hence, it is concluded, by virtue of Cauchy's theorem, that the asymptotic expansion $\left(13.2^{*}\right)$ is uniformly convergent for $\|V\|<b_{0}^{\prime \prime}$. Therefore, owing to the Proposition 13.1, the power series (13.2) also converges for $\|U\|<b_{0}^{\prime \prime}$ and represents a solution of the equation (13.1) (i.e. the equation (A.14)). We define the $n$ vector $P^{(0)}(u)$ by $S(u)$.

\section{Determination of the mavectors $P^{(k)}(u)$.}

Since the method to determine the functions $P^{(k)}(u)$ for $k \geqq 2$ is quite the same as that for the $n$-vector $P^{(1)}(u)$, we discuss the case for $k=1$ only.

14. 1. As we have just seen, the vector function $P^{(0)}(u)$ is expressible as uniformly convergent power series of $u$ for $\|u\|<b_{0}^{\prime \prime}$. Hence, the equation (A.17) ${ }^{1}$ appearing in Introduction can be written as

$$
x P^{\prime}=-P+F(U) P+R(U),
$$

where the $n$-by-n matrix $F(u)$ and the $n$-vector $R(u)$ are functions holomorphic and bounded in $u$ for $\|u\|<b_{0}^{\prime \prime}$ and, especially, $F(0)=0$. Moreover, this equation admits a formal solution of the form

$$
P \sim \sum_{\mathcal{K}} P_{\mathcal{K}}^{(1)} U \mathcal{K}
$$

Let $x=\tilde{x}(w), u=\tilde{U}(w)$ be the holomorphic solution of the differential equations $\left(R^{\prime}\right)$ in Section 5 , i. e.

$$
w^{2}(\mathscr{M} \cdot X(u)) \frac{d x}{d w}=x, \quad w(\mathscr{H} \cdot X(u)) \frac{d u}{d w}=\mathbb{1}_{n}(X(u)) u
$$

with 


$$
X(u)=\alpha+\sum_{\mathscr{H} \in \Phi^{S}} \beta_{\mathscr{H}^{\prime}} \mathfrak{H}^{\mathscr{H}}
$$

satisfying the initial condition $\tilde{x}\left(w^{0}\right)=x_{0}, \tilde{U}\left(w^{0}\right)=u$.

Here, $x_{0}$ and $u^{0}$ are arbitrary values such that

$$
\left|x_{0}\right|<a_{0}^{\prime \prime}, \quad\left\|u i^{0}\right\|<b_{0}^{\prime \prime}, \quad\left(u^{0}\right) \mathscr{M} \in \mathfrak{D}\left(c_{0}^{\prime \prime}\right),
$$

and the quantity $r w^{0}$ is defined by

$$
w^{0}=\left(u^{0}\right) \pi
$$

where the domain $\mathfrak{D}\left(c^{\prime \prime}\right)$ is defined by (6.1) with $c_{0}=c_{0}^{\prime \prime}$. Then, as we have already seen, we have the identities

$$
\widetilde{U}(w) \mathcal{I} \equiv \mathcal{U}, \quad U\left(x, x_{0}, \imath^{0}\right) \equiv \widetilde{U}\left(\tilde{x}^{-1}(x)\right) .
$$

We can assert that:

Proposition 14.1. The formal power series

$$
P \sim \sum_{\mathcal{K}} P_{\mathcal{K}}^{(1)} \tilde{U}(\tau) \mathcal{K}
$$

is a formal solution of the differential equation

$\left(14.1^{*}\right) \quad w^{2}(\mathcal{M} \cdot X(\tilde{U})) \frac{d P}{d w}=-P+F(\tilde{U}) P+R(\widetilde{U}),(\tilde{U} \equiv \tilde{U}(w))$.

Conversely, the solution $g(\widetilde{U}(w))$ of the equation (14.1*) becomes a solution of the equation (14.1) if re replace $\widetilde{U}\left(w^{\prime}\right)$ by $U\left(x, x_{0}, u^{0}\right)$.

The proof of this proposition is almost exactly the same as that of the Proposition 13.1.

14. 2. We can prove the following assertion:

The differential equation (14.1*) possesses a solution $P=S(\widetilde{U}(w))$ which admits the asymptotic expansion $\left(14.2^{*}\right)$ as $\tilde{U}(w)$ tends to 0 in the domain

$$
\|u\|<b_{0}^{\prime \prime}, \quad u \mathfrak{M} \in \mathscr{D}\left(c_{0}^{\prime \prime}\right) .
$$

In order to prove this assertion, we make a transformation of the form

$$
P=p+P^{N}\left(\tilde{U}\left(w^{\prime}\right)\right)
$$

where 


$$
P^{N}(u)=\sum_{|\mathcal{K}|<N} P_{\mathcal{K}}^{(1)} u^{\mathcal{K}}
$$

If we notice that $(\mathscr{H} \cdot X(u))=(\mathscr{M} \cdot \alpha)+O(u)$, the transformed equation can be written as

$$
w^{2} \frac{d p}{d w}=-\frac{1}{(\mathscr{M} \cdot \alpha)} p+F_{1}(\tilde{U}) p+R_{1}(\tilde{U})
$$

with

$$
F_{1}(u)=\frac{1}{(\mathscr{M} \cdot X(u))} F(u)+\frac{\sum(\mathscr{M} \cdot \beta \mathscr{H}) u^{\mathscr{H}}}{(\mathscr{M} \cdot \alpha)(\mathscr{M} \cdot X(u))} \mathbf{1}_{n}
$$

where $R_{1}(u)$ is an $n$-dimensional column vector function holomorphic and bounded in $u$ for $\|u\|<b_{0}^{\prime \prime}$.

Since this equation admits a formal solution

$$
p \sim \sum_{|\mathcal{K}| \geqq N} P_{\mathcal{K}}^{(1)} \tilde{U}(w) \mathcal{K},
$$

we can prove that the inequalities

$$
\left\|F_{1}(u)\right\| \leqq A, \quad\left\|R_{1}(u)\right\| \leqq B_{N}\|u\|^{N}
$$

are satisfied for $\|u\|<b_{0}^{\prime \prime}$. Here, $A$ is independent of $N$ and $u$, while $B_{N}$ may depend on $N$.

It should be noticed that, since $F_{1}(0)=0$, the quantity $A$ can be taken as small as we want.

We apply a further transformation of the form

$$
p=q \exp \Lambda(w), \quad \Lambda(w)=1 /(\mathscr{M} \cdot \alpha) w .
$$

Then, we have the equation

$$
\frac{d q}{d w}=w^{-2}\left\{F_{1}(\tilde{U}) q+R_{1}(\tilde{U}) e^{-\Lambda(w)}\right\} .
$$

14. 3. Let $\Gamma_{w 0}^{*}$ be the same as that appearing in Theorem 6.1. Then, we can prove the following:

Proposition 14.2. Let $s$ be the length of this curve measured from the origin to the point $w$. Then, we have the inequality

$$
\frac{d}{d s}\left(\|\tilde{U}\|^{N} e^{-\operatorname{Re} \Lambda(w)}\right) \geqq \frac{\sin \boldsymbol{\delta}_{0}}{2|(\mathcal{M} \cdot \boldsymbol{\alpha})||w|^{2}}\left(\|\widetilde{U}\|^{N} e^{-\operatorname{Re} \Lambda(w)}\right)
$$

on the path $\Gamma_{w 0}^{*}$. 
Proof. Since

$$
w^{2} \frac{d}{d w} e^{\frac{1}{(\mathscr{M} \cdot \alpha) w}}=\frac{1}{(\mathscr{M} \cdot \alpha)} e^{-\frac{1}{(\mathscr{M} \cdot \alpha) w}},
$$

the differential equation satisfied by the function $\exp \left(-\frac{1}{(\mathscr{H} \cdot \boldsymbol{\alpha}) \boldsymbol{w}}\right)$ coincides with that for the function $\tilde{x}(w)$ if we put there $\alpha^{*}=0$. Hence, as we have already seen in Theorem 6.1, we have

$$
\begin{gathered}
\frac{d}{d s}\left(\|\widetilde{U}\|^{N} e^{-\operatorname{Re} \frac{1}{(\mathscr{M} \cdot \alpha)} \bar{w}}\right) \geqq \frac{\left(N|w|\|\alpha\|^{\prime}+1\right) \sin \delta_{0}}{2|(\mathscr{M} \cdot \alpha)||w|^{2}}\left(\|\widetilde{U}\|^{N} e^{-\operatorname{Re} \frac{1}{(\mathscr{M} \cdot \alpha) w}}\right) \\
\geqq \frac{\sin \delta_{0}}{2|(\mathscr{M} \cdot \alpha)||w|^{2}}\left(\|\tilde{U}\|^{N} e^{-\operatorname{Re} \frac{1}{(\mathscr{M} \cdot \alpha) w}}\right) .
\end{gathered}
$$

Using this inequality, we can establish the following proposition:

Proposition 14.3. The differential equation (14.8) admits one and only one solution $q=\Phi^{N}(\widetilde{U}(w))$, where the n-rector $\Phi^{N}(u)$ is a function holomorphic and bounded in $u$ for

$$
\|u\|<b_{N}^{\prime \prime}, \quad u M l \in \mathfrak{D}\left(c_{N}^{\prime \prime}\right)
$$

and satisfying there the inequality

$$
\left\|\Phi^{N}(u)\right\| \leqq K_{N}\|u\|^{N} e^{-\operatorname{Re} \Lambda(w), \quad(w=u \mathscr{M}),}
$$

$K_{N}$ being a certain positive constant.

Proof. For the proof of this proposition, let $\mathfrak{F}=\{\varphi(u)\}$ be the family of the $n$-vectors $\varphi(u)$, which are functions holomorphic and bounded in $u$ for the domain $(14.10)_{N}$ and satisfying there the inequality

$$
\|\varphi(u)\| \leqq K_{N}\|u\|^{N} \exp \left(-\operatorname{Re} \Lambda\left(u^{M}\right)\right) .
$$

We define the $n$-vector $\bar{\varphi}(u)$ by the formula

$$
\bar{\varphi}\left(u^{0}\right)=\int_{\Gamma_{l_{0}}^{*}} w^{-2}\left\{F_{1}(\tilde{U}(w)) \varphi(\tilde{U}(w))+R_{1}(\tilde{U}(w)) e^{-\Lambda(w)}\right\} d w
$$

where $u^{0}$ is an arbitrary point in the domain $(14.10)_{N}$ and

$$
w^{0}=\left(u^{0}\right) \mathscr{M} .
$$


Then, the mapping $\mathfrak{I}$ is defined as follows:

$$
\varphi(u) \stackrel{\mathfrak{I}}{\longrightarrow} \bar{\varphi}(u) .
$$

The proof of the Proposition 14.3 is almost exactly the same as that of the Theorem 9.1, which was given in Sections 9 11, except for the proof of the inequality

$$
\left\|\bar{\varphi}\left(u^{0}\right)\right\| \leqq K_{N}\left\|u^{0}\right\|^{N} e^{-\operatorname{Re} \Lambda\left(w^{0}\right)} .
$$

Therefore, we discuss the inequality (14.14) only.

By the inequalities $(14.12)_{N}$ and (14.6), we have

$$
\left\|\bar{\varphi}\left(u^{0}\right)\right\| \leqq \int_{0}^{s_{0}}\left(A K_{N}+B_{N}\right)\|\tilde{U}(w)\|^{N} e^{-\mathrm{Re} A(w)}|w|^{-2} d s,
$$

where $s_{0}$ is the length of the curve $\Gamma_{w 0}^{*}$.

By definition of the curve $\Gamma_{w 0}^{*}$ in Section 7 , if $s$ is sufficiently small, we have $s=|w|$ and $\|\widetilde{U}(w)\|$ is a bounded function whose nature is clarified by the relation (11.5), while the function $\exp (-\operatorname{Re} \Lambda(w))$ tends exponentially to zero with $s$. From this we see that the integral appearing in the right-hand member of the above inequality and, consequently, the integral (14.13) is uniformly convergent.

On the other hand, by using the inequality (14.9), we have immediately the inequality

$$
\left\|\bar{\varphi}\left(u^{0}\right)\right\| \leqq \frac{2 \mid(\mathscr{M} \cdot \alpha) !\left(A K_{N}+B_{N}\right)}{\sin \delta_{0}}\left\|u^{0}\right\|^{N} e^{-\mathrm{Re} \Lambda\left(w^{0}\right)} .
$$

Therefore, to obtain the inequality (14.14), it is sufficient to show that we have

$$
2|(\mathscr{M} \cdot \alpha)|\left(A K_{N}+B_{N}\right) \leqq K_{N} \sin \delta_{0} .
$$

However, as we have already remarked, we can assume, without loss of generality, that

$$
4|(\mathscr{M} \cdot \alpha)| A<\sin \delta_{0}
$$

Then, by taking $K_{N}$ sufficiently large, we have the inequality (14.15).

14.4. By virtue of the Proposition 14.3, we see that 


$$
\left.P^{N}(\widetilde{U}(w))+\Phi^{N}(\widetilde{U}(w)) e^{\Lambda(\widetilde{U}(w)} \mathscr{M}\right)
$$

is a solution of the equation $\left(14.1^{*}\right)$ and is independent of $N$. We denote this expression by $S(\tilde{U}(w))$. Then, the $n$-vector $S(\tilde{U}(w))$ admits the asymptotic expansion $\left(14.2^{*}\right)$ as $\widetilde{U}(w)$ tends to 0 in the domain (14.3) with $b_{0}^{\prime \prime}=\sup b_{N}^{\prime \prime}$ and $c_{N}^{\prime \prime}=\sup c_{N}^{\prime \prime}$.

Owing to the Proposition 14.1 the $n$-vector $P^{(1)}(u)$ defined by $S(u)$ is a solution of our problem.

14. 5. The determination of the $n$-vectors $P^{(k)}(u)$ for $k \geqq 2$ can be carried out in quite a similar way. The only difference between the case for $k \geqq 2$ and the case for $k=1$ is as follows: For $k \geqq 2$, the $n$-vector $R^{(k)}(u)$ appearing in (A.17) in Introduction depends actually on the $n$-vector $P^{(1)}(u)$. Hence, the vector function corresponding to the $n$ vector $R_{1}(u)$ appearing in (14.5) admits an expansion not convergent but asymptotic in powers of $u$ for the domain (14.3).

\section{REFERENCES}

M. Hukuhara [1]: Sur les points singuliers d'une équation différentielle ordinaire du premier ordre, I. Mem. Fac. Eng. Kyûshû Imp.Univ., 8 (1937), 203-247.

[2]: Pri la elvolvado de Solvo de diferentialaj ekvacioj en la chirkauajho de ilia singula punkto. Mem. Fac. Sci. Kyûshû Imp.Univ., 4 (1949), 1-7.

[3]: Renzokuna Kansu no Zoku to Syazo. Mem. Fac. Sci. Kyûshû Univ., Ser. A, 5 (1950), 61-63.

[4]: Équations différentielles ordinaires du premier ordre dans le champ complex (with T. Kimura and Mme. T. Matuda). Publ. Math. Soc. Japan, 7 (1961).

M. Iwano [1]: Intégration analytique d'un système d'équations différentielles non linéaires dans le voisinage d'un point singulier, I. Ann. Mat. Pura Appl., Ser. 4, 44 (1957), 261-292.

[2]: On a singular point of Briot-Bouquet type of a system of two ordinary nonlinear differential equations. Publications of Research Inst. Math. Sci. Kyoto Univ.), Ser. A, 2 (1966), 17-115.

[3]: Convergent solution of ordinary nonlinear differential equations.

Funkcialaj Ekvacioj, 9 (1966), 273-285.

Mathematics Research Center

United States Army

University of Wisconsin

and

Department of Mathematics

Tokyo Metropolitan University 
